
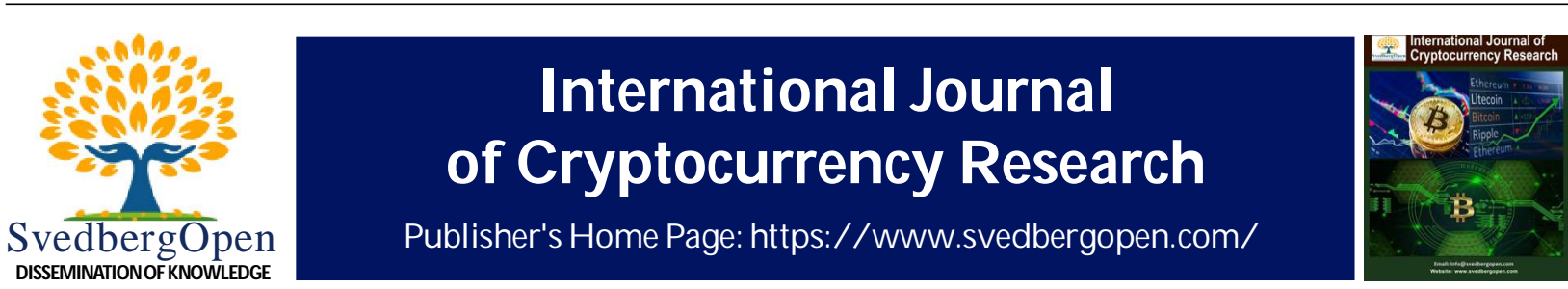

Research Paper

Open A ccess

\title{
Blockchain Technology and Intellectual Property - A Basic Introduction
}

Julia Hugendube $1^{1^{*}}$

${ }^{1}$ Senior Associate, CMS, Kranhaus 1, Im Zollhafen 18, 50678 Köln, Germany. E-mail: julia.hugendubel@cms-hs.com

Article Info

Volume 1, Issue 1, December 2021

Received : 12 September 2021

Accepted : 21 November 2021

Published : 05 December 2021

doi: 10.51483/IJCCR.1.1.2021.5-25

\begin{abstract}
Blockchain technology is predicted to have a major impact on the Intellectual Property (IP) ecosystem. More and more projects are being launched, both in the public and private sector. The World Intellectual Property Organization (WIPO) has built up a Blockchain Task Force and is preparing a new WIPO Standard to encompass all types of IP rights and the entire IP lifecycle ${ }^{2}$; the German Government published a strategy paper on blockchain with a chapter on applications in the creative arts sector ${ }^{3}$; a European Blockchain Service Infrastructure is being built up ${ }^{4}$; the European Union Intellectual Property Office (EUIPO) established an Anti-Counterfeiting Forum as part of the broader EU strategy to create a blockchain ecosystem ${ }^{5}$ and now has its own blockchain for trademarks and designs in the $\mathrm{EU}^{6}$. Furthermore, LVMH, with brands such as Louis Vuitton, developed its own blockchain to track luxury goods ${ }^{7}$; Kodak started a blockchain initiative for image rights management ${ }^{8}$; music and film streaming are offered on blockchain platforms ${ }^{9}$; sports clubs discuss microlicensing of their IP rights ${ }^{10}$; digital fashion is created for distribution using blockchain ${ }^{11}$. Moreover, news abounds of Non-Fungible Tokens (NFTs) representing basically everything imaginable — both digital and physical—in the IP ecosystem, to track the origin of art and all manner of products. However, interestingly enough, as early as 2012 a whitepaper was published linking the idea of smart property by Nick Szabo and digital collectibles to blockchain and at the same time introducing the first kind of NFTs, colored coins ${ }^{12}$.
\end{abstract}

Keywords: Blockchain technology, Intellectual Property ecosystem, WIPO, NFTs

(C) 2021 Julia Hugendubel. This is an open access article under the CC BY license (https://creativecommons.org/licenses/by/4.0/), which permits unrestricted use, distribution, and reproduction in any medium, provided you give appropriate credit to the original author(s) and the source, provide a link to the Creative Commons license, and indicate if changes were made.

\footnotetext{
* Corresponding author: Senior Associate, CMS, Kranhaus 1, Im Zollhafen 18, 50678 Köln, Germany.

E-mail: julia.hugendubel@cms-hs.com

2 WIPO Report by the Blockchain Task Force (November 2020) 3 <www.wipo.int/edocs/mdocs/cws/en/cws_8/cws_8_15-main1.pdf> accessed on July 20, 2021; Julia Hugendubel (December 2020). Blockchain Standard for IP Offices: The WIPO Blockchain Projects. <https://ipkitten.blogspot.com/2020/12/blockchain-standard-for-ip-offices-wipo.html> accessed $20 \mathrm{July} 2021$.

3 Blockchain Strategy of the German Government (2019). <www.blockchain-strategie.de/BC/Navigation/DE/Home/home.html> accessed on July 20, 2021. <https://ec.europa.eu/cefdigital/wiki/display/CEFDIGITAL/EBSI> accessed on July 20, 2021.

<https://euipo.europa.eu/ohimportal/en/web/observatory/blockathon> accessed on July 20, 2021.

<https://euipo.europa.eu/ohimportal/en/news/-/action/view/8662923> accessed on July 20, 2021.

Ian Allison (2019). Louis Vuitton Owner LVMH Is Launching a Blockchain to Track Luxury Goods. <www.coindesk.com/louisvuitton-owner-lvmh-is-launching-a-blockchain-to-track-luxury-goods > accessed on July 20, 2021.

8 <www.kodakone.com/kodak-and-wenn-digital-partner-to-launch-major-blockchain-initiative-and-cryptocurrency/> accessed on July 20, 2021. E.g. <https://ujomusic.com/, https://soundac.io/, www.thetatoken.org/, https://cinemadrom.com/> accessed on July 20, 2021.

10 <www.bvb.de/Partner/News/SPORTFIVE-vermittelt-Blockchain-Partnerschaft-zwischen-dem-B VB-und-Liquiditeam>; Thomas Euler, The Tokenization Playbook for the Sports Industry (2019). <https://medium.com/liquiditeam/the-tokenization-playbook-for-thesports-industry-guide-blockchain-busines-87da13d33dfe> accessed on February 20, 2021.

11 <https://www.lukso.network/> accessed on July 20, 2021.

$1^{12}$ Meni Rosenfeld (2012). Overview of Colored Coins. <https://bit.ly/3yBRDiF> accessed on March 23, 2021.
}

2790-1386/@ 2021. Julia Hugendubel. This is an open access article distributed under the Creative Commons Attribution License, which permits unrestricted use, distribution, and reproduction in any medium, provided the original work is properly cited. 


\section{Introduction}

The developments in the blockchain space, including in the area of IP, are progressing at a rapid pace, both from a technological and a value perspective. For example, the value of crypto art traded on blockchain from 2018 to 2020 was estimated at about $\$ 15 \mathrm{mn}$ (with about $\$ 8.2 \mathrm{mn}$ worth of crypto artwork in December $2020^{13}$ ). In March 2021, the most expensive piece of art linked to an NFT in history was sold by Mike Winkelmann (Beeple) for 42,329.453 Ether, at the time worth $\$ 69,346,250 \mathrm{mn} \cdot{ }^{14}$ The NFT marketplace OpenSea has set and then beat daily records several times, reaching a new peak of $\$ 322,982,301 \mathrm{mn}$ of trading volume on 29 August $2021 . .^{15}$

Nevertheless, many applications for IP matters are still in their infancy. The reason for this might lay, aside from the rather complex technological details, in uncertainties about their regulation and legal standing in court, such as the recognition of a legal binding smart contract.

Therefore, the article introduces the very basic features of blockchain technology and blockchain-based IP applications. The paper then dives into concrete IP use cases that are currently offered and developed on the market. It also gives a general overview of opportunities and existing challenges for the IP ecosystem. The legal perspective is mainly a German and European one.

\section{Blockchain Features for IP Matters}

Blockchain technology is a decentralised, distributed database in a peer-to-peer network using cryptography for its transactions. Bitcoin and Ethereum are just two popular examples of a growing number of blockchain platforms.

\subsection{The Basics of Blockchain Technology}

The features of blockchains such as Ethereum not only allow the transfer of money, but of anything that can be represented by data information, such as an IP right.

In principle, there are three categories, namely, public, private and consortium blockchains. A public blockchain has a public network that is generally programmed to be accessible for everyone, meaning permissionless, although its access may also be subject to certain conditions and thus depends on the permission of the algorithm. A private blockchain, on the other hand, is launched by a private entity and often restricted to selected users. Nevertheless, they can be both permissioned and permissionless. Permissioned blockchains use an access control layer in their protocol. The consortium blockchain is a mixture of permissioned and public blockchains; its infrastructure is still decentralised, but the consensus is managed by a set of participants. ${ }^{16}$

Blockchain can be seen as a generic term, describing the function of this technology: Transactions are bundled together to be added as a block to the previous blocks, thus creating a chain of blocks. Blocks are used to store the transactions and other required data to successfully operate the blockchain. A new block is added if the validation protocol of the respective blockchain is fulfilled. Every blockchain starts with a block - block 0 - that is the secure root on which a trusted chain is built. The longest chain of blocks that is validated and then added after block 0 is considered to be the current blockchain status. ${ }^{17}$

\section{a. Cryptography}

Blockchain technology works with asymmetric cryptography in different ways, such as to access, transfer, and secure data. $^{18}$

Two different tools are needed to access information on the blockchain: a private key and a public key. The private key is only in the possession of the individual user, comparable to a bank account PIN. This is why the private key is also called a secret key. The public key can be compared to a bank account number. A decryption of the transaction is only

13 <https://www.statista.com/statistics/1221400/nft-sales-revenue-by-segment/>; <https://decrypt.co/52995/8-2-million-worth-ofcrypto-artwork-sold-in-december> accessed on July 20, 2021.

$14<$ https://www.instagram.com/p/CLXQ3WKgwuw/?utm_source=ig_embed> accessed on July 20, 2021.

$15<$ https://dune.xyz/rchen8/opensea> accessed on August 29, 2021.

16 Vitalik Buterin (2015). On Public and Private Blockchains, Ethereum Blog. <https://blog.ethereum.org/2015/08/07/on-public-andprivate-blockchains/> accessed on July 20,2021; Omar Dib (2018). Consortium Blockchains: Overview, Applications and Challenges. <https://bit.ly/366zehz> accessed on July 20, 2021.

17 E.g. Vitalik Buterin (2013). Ethereum White Paper, 9. <https://ethereum.org/en/whitepaper/> accessed 20 July 2021; Markus Kaulartz, Die Blockchain-Technologie, CR 2016, 474, 476; Joseph J. Bambara/Paul R. Allen et al. (2019). AI, IoT and the Blockchain. ch. 2, 3 (25).

${ }^{18}$ Daniel Drescher (2018). Blockchain Básico, 58, 59. 
possible together with the public and the private key. ${ }^{19}$ Only the sender's private key is compatible with their public key and only with the combination of both can the sender provide the signature required to authorise a transaction from their account. ${ }^{20}$

Information on a blockchain is stored in cryptographic hashes. A hash is created by a 'hash function', i.e., a small computer program that 'transforms any type of data into numbers and letters' ${ }^{21}$ of a fixed size, whatever the size of the data entered. These cryptographic hash functions rapidly deliver hash values for any type of data. Any song or movie, for example, can be represented by a hash of the same size, whether it lasts three minutes or three hours.

The main 'characteristic' of a hash function is its 'determination', i.e., the same data will always produce the same hash, but even a slight change will result in a different hash. This is also why they are said to be collision resistant; it is virtually impossible for the same hash to be generated with other data. Furthermore, a cryptographic hash is 'unidirectional', i.e., it is not possible to decrypt the hash. ${ }^{22}$

A hash value depends on the selected hash function, such as SHA-256. SHA-256 generates a hash length of 32 bytes (256/8). ${ }^{23}$ The hash generated with SHA-256 for "Intellectual Property" is:

\section{CEEF38A020E129CA4EB9B2A3088FCFFE2F12B400E00894C3EE28C20D779F4F6}

In contrast, the hash for the same term written in small letters - "intellectual property" - is:

\section{E15FDCB4BDD3495441046B6997F84E389E31990312BFB0647726B0E9503CA5024}

This demonstrates how any change of data leads to a different hash value. If there is any deviation in the hash value then it is proven that the underlying data is differing. ${ }^{25}$ Because of its unique representation, a hash value is often compared to the 'digital fingerprint' of the underlying digital information.

\section{b. Block(-Chain) Content}

The content of a blockchain is called the ledger. Blockchains are therefore also referred to as Distributed Ledger Technology (DLT), although not every decentralised ledger network need be a blockchain ${ }^{26}$.

The ledger is used to maintain information about ownership of anything that is represented on the blockchain, similar to the ledger of a company's account book. The ledger represents the status of all accounts and all performed transactions. Essential for the IP ecosystem is that the data represented on a blockchain is not limited to cryptocurrencies; all different types of data can be deployed on a blockchain. ${ }^{27}$ Examples are IP assets such as art, music, movies but also supply chains of IP-protected goods. Even trademarks can be included in a smart contract so that they are visible in the public transaction and in the account of the respective user.

In an IP transaction, each block generally contains the hash of the information relating to the transactions (timestamp, sender, recipient, transaction fee and the IP asset that is transferred), the hash of the previous block and information for securely and efficiently encoding data (Merkle tree root hash). Additional information is added depending on the protocol. The chain of blocks is created because from each block, a hash value is created and each following block after block 0 includes the hash from the previous block ${ }^{28}$. Thus, any change in the ledger will lead to a different hash value and will therefore make the other following hash values "false", i.e., not compliant with the previous hash value. ${ }^{29}$

To participate in a public blockchain, e.g., buying and making other transfers with crypto-assets such as NFTs, it is generally sufficient to have a wallet. There are different types of wallets: software and hardware wallets. Depending

\footnotetext{
Kaulartz, in: Briner/Funk, DGRI Jahrbuch 2017, 1. Ed. (2018), Blockchain und Smart Contracts, para. 9.

Kaulartz (2016). Die Blockchain-Technologie, CR, 474, 476.

The numbers and letters represent the numbers of a hexadecimal system $(0-9$ and a-f).

Drescher (2018). Blockchain Básico. 83, 85 with further references; <https://mathworld.wolfram.com/HashFunction.html> accessed on July 20, 2021.

23 Kaulartz (2018). op. cit., para. 8.

24 <https://passwordsgenerator.net/sha256-hash-generator/> accessed on July 20, 2021.

25 Kaulartz (2016). op. cit., CR, 474, 475.

26 E.g. https://hedera.com/ accessed on July 20, 2021. According to the Proposal for a Regulation of the European Parliament and of The Council on Markets in Crypto-assets, Article 3 no. 1 (2) 'distributed ledger technology' or 'DLT' means a type of technology that support the distributed recording of encrypted data, https://eur-lex.europa.eu/resource.html?uri= cellar:f69f89bb-fe54-11 eab44f-01aa75ed71a1.0001.02/DOC_1\&format=PDF accessed on July 20, 2021.

27 <http://iprhelpdesk.eu/ip-highlights/ip-special-blockchain/blockchain-in-a-nutshell> accessed 20 July 2021.

28 So-called "block header".

29 To add a new block to the existing blockchain, the hash value of the block header has to be verified if it combines with the hash values of the previous blocks. This is done to ensure that nobody can carry out a transaction without having rightful ownership of
} the item to be transacted. In detail, Buterin (2013). op. cit., 9f., 19, 22. 
on jurisdiction, a user has to go through a Know Your Customer (KYC), Anti-Money Laundering (AML) $)^{30}$ and Combating the Financing of Terrorism (CFT) procedure before using the wallet. Wallets generally allow the user to arbitrarily create new accounts for transactions, i.e., each account in a wallet can send and receive transactions, similar to a bank account.

A new block is added depending on the blockchain's protocol. Strict compliance with the block validation protocol in public blockchains is ensured by a consensus mechanism. The consensus mechanism is the reason why this technology can work as a trusted system without trust-building intermediaries. Validation prevents fraudulent transactions and the "double-spending" of cryptocurrency or other assets on the respective blockchain.

The most well-known consensus mechanism is the 'proof of work' as it is used in the Bitcoin blockchain. To add a package of transactions to the blockchain (block), a computing task must be solved to find a particular number of data (so-called "nonce" ${ }^{31}$ ). The participating computers that validate the transactions are called "nodes". The chances of a node solving the computer task increase depending on computing power. The node that solves the exercise first will be rewarded according to the blockchain protocol. Because the reward is a newly issued cryptocurrency, similar to additional printed Fiat currency ${ }^{32}$, the process is called "mining" and the nodes are called "miners". ${ }^{33}$ Additionally, a transaction fee is payed as a reward for the miner, to ensure that the transaction concerned is included in a block. For example, if someone wants to transfer an IP right to a third party on a public blockchain, a transaction fee motivates miners to include the transaction in the next block to receive the transaction fee as a reward.

Another possible consensus mechanism is the 'proof of stake' as it will be used for Ethereum $;{ }^{34}$ however, there are various other consensus mechanisms, such as proof of capacity, proof of history or proof of authority. ${ }^{35}$ Permissioned blockchains might therefore use a more "simple" mechanism to add transactions to their chain.

\section{c. Characteristics of Blockchains}

The original idea for blockchain was to offer a solution for the so-called double spending problem by creating a system that replaces the need to rely on a trusted third party to act as an intermediary, because the technology itself is trusted. ${ }^{36}$ Any hacker wanting to manipulate a transaction in the blockchain would have to reprogram all following hash values of the blockchain or the nonce value in the block that leads to the required hash value. This is the main reason why the ledger of a-sufficiently distributed-blockchain is generally seen as 'immutable'. ${ }^{37}$ Generally, it is only possible to append data, but not to delete it. ${ }^{38}$

Furthermore, because assets deployed on a blockchain are not sent as such, but their access rights, they cannot be spent twice. This is not only relevant for monetary spending, but also in the field of IP, for example to identify ownership or the chain of rights. Together with the unlikeliness of any change in the ledger, blockchains are therefore seen as highly 'reliable' 39 . In addition, because any one in a permissionless (public) blockchain can verify the transaction chain and its consistency, the blockchain is regarded as 'transparent'. This means, however, that the address of a transaction in a public blockchain, which refers to the public key, as well as the sent value are generally open to the public. ${ }^{40}$

30 www.reddit.com/r/CoinBase/comments/d96g2n/what_are_amlkyc_for/> accessed on July 20, 2021.

31 The "nonce" is the data that has to be found to create a hash of the block that is smaller as defined by the protocol and en-sures that each block can only be processed once, ibid $13 \mathrm{f}$.

32 Fiat currency "is money that is declared legal tender and issued by a central bank but, unlike representative money, cannot be converted into, for example, a fixed weight of gold." <www.ecb.europa.eu/explainers/tell-me-more/html/what_is_money.en.html> accessed on July 20, 2021.

33 Buterin (2013). Ethereum White Paper, 7.

34 <https://docs.ethhub.io/ethereum-roadmap/ethereum-2.0/proof-of-stake/>. The right to validate a block depends here on the node's cryptocurrency. A node is only allowed to validate if it has a certain amount of money on its account. The higher the locked amount, the better the chance that the node can mine the next block. To secure this procedure, the assets of the validating account that are "at stake" are inaccessible for a certain time period until it can be assured that the validation was correct. If the mining node makes a mistake, it will lose its stake, i.e. the locked amount <https://eth.wiki/en/concepts/proof-of-stake-faqs> accessed 20 July 2021.

35 Bambara/Allen et al. (2019). op. cit, ch. 2, (4, 21).

36 In detail, Satoshi Nakamoto (2008). Bitcoin: A peer-to-peer Electronic Cash System. 2, 4 <https://bitcoin.org/bitcoin.pdf> accessed on July 20, 2021

37 Hye-young Paik et al. (2019). Analysis of Data Management in Blockchain-Based Systems: From Architecture to Governance. IEEE Access <https://www.researchgate.net/publication/338144930_Analysis_of_Data_Management_in_BlockchainBased_Systems_From_Architecture_to_Governance> accessed on July 20, 2021.

38 Kaulartz (2018). op. cit., para. 6.

39 Ibid

40 Nevertheless, there are tools to avoid this traceability, such as cryptocurrency tumblers or other services. However, these options are generally not (yet) implemented by default in the current public blockchains applications such as for Bitcoin or Ethereum. 
Moreover, since the blockchain is saved on any computer participating as a full node, it enables high, fast and paperless data availability. Thus, blockchain technology can also be compared to a database that allows an incredible amount of data to be saved and transferred. Through the use of smart contracts, all kinds of transaction processes can then be automated, such as payment or licensing to enhance efficiency. ${ }^{41}$

\subsection{Smart Contracts}

Smart contracts are necessary to trigger automatic transactions between blockchain addresses. ${ }^{42}$ In general, it is possible to use smart contracts as a medium to execute legally binding transactions. The crucial factor is what the parties intended. ${ }^{43}$ However, smart contracts are not necessarily contracts. Introduced by Nick Szabo in the $90 \mathrm{~s}^{44}$ and linked to blockchain technology by Vitalik Buterin in $2013,{ }^{45}$ the denomination simply refers to self-executing computer codes. Consequently, they can be used for any condition that can be programmed. ${ }^{46}$ If certain conditions are fulfilled, such as the occurrence of an event or a violation of a contractual obligation, the smart contract automatically triggers the intended consequence. ${ }^{47}$

Though smart contracts can be programmed on any computer or platform outside of a blockchain, the potential of a smart contract increases when deployed on a blockchain. ${ }^{48}$ In combination with a blockchain, no reliance on a central server is required and the distribution is safeguarded by the data structure of the chain as well as its encrypted transactions. Without using a blockchain, smart contracts face the same hurdles as centralised databases; in particular, if the database fails, the smart contract will fail, too, and since any change to the smart contract is difficult to track, it can easily be changed or manipulated. ${ }^{49}$

Smart contracts on blockchain are already used for various IP use cases, such as distributing music. People that listen to the music automatically pay for it through their (blockchain) account. The idea is that no intermediaries are required and artists directly receive their royalties. ${ }^{50}$ Smart contracts are in general programmed with conditions such as "if - then - else". For instance: If Alice wants to listen to the song "Hello World!" by Bob then she has to pay a certain amount of royalties to Bob. The smart contract is, for example, programmed with the arguments "verification of the song", "verification of the requested use", "verification of the amount of royalties", "verification of the signature for the respective account". If one of the requested pieces of information does not match the conditions of the smart contract, an error is returned and the transaction is not completed. No intermediary is needed to give Alice access to the music or to send Bob his royalties. Each song entry is programmed with instructions on who will receive what percentage of the paid royalties. The smart contract is self-enforcing.

Other licences or IP transactions, such as those involving copyrights, trademarks, designs or patents can likewise be programmed with a smart contract. ${ }^{51}$ As such, individual blockchain applications and smart contracts might be copyrightprotected as computer programs (Art. 10 of the TRIPS Agreement in conjunction with Art. 2 of the Berne Convention and Art. 4 WIPO Copyright Treaty; Art. 1 EU Directive 2009/24/EC, §§ 69a ff. German Copyright Act, implementing the EU Directive 2009/24/EC).

\subsection{Token}

An IP or any other asset on a blockchain can be represented by a cryptographic token ${ }^{52}$. Tokens on a blockchain are managed by smart contracts and represent values. Only those who have the private key can use and dispose of the token. $^{53}$

41 Emmanuelle Ganne (2018). Can Blockchain revolutionize international trade? <www.wto.org/english/res_e/publications_e/ blockchainrev18_e.htm> accessed on July 20, 2021.

42 Bambara/Allen et al. (2019). op. cit., ch. 2, (4).

43 Markus Kaulartz/Jörn Heckmann (2016). Smart Contracts - Anwendungen der Blockchain-Technologie, CR, 618, 621.

44 Smart Contracts: Building Blocks for Digital Markets (1996). <www.fon.hum.uva.nl/rob/Courses/InformationInSpeech/CDROM/ Literature/LOTwinterschool2006/szabo.best.vwh.net/smart_contracts_2.html>; Smart Contracts: Formalizing and Securing Relationships on. Public Networks, First Monday, Vol 2, No 9 (1997) <https://bit.ly/3k5MGaq> accessed on July 20 , 2021.

45 Buterin (2013). op. cit.

46 Each blockchain protocol requires a different program language, for Ethereum even a new program language was developed called Solidity.

47 Nicolas Hohn-Hein/Günter Barth (2018). Immaterialgüterrechte in der Welt von Blockchain und Smart Contract, GRUR, 1089 , 1093.

48 Kaulartz (2018). op. cit., para. 1.

49 Emmanuelle Ganne (2018). op. cit.

50 <https://docs.soundac.io/> accessed on July 20, 2021.

51 In detail, chapter II. 2. of this article.

52 Another possibility to represent IP on a blockchain are verifiable credentials, using, e.g., JSON, or simply to deploy the hash of the IP medium on the blockchain.

53 <https://blockchainhub.net/tokens/> accessed on July 20, 2021. 
As cryptographic tokens enable a unique digital representation, they make it possible to represent and transfer any ownership or right of an asset. Consequently, a token transaction from Alice to Bob means that Alice can no longer dispose of the token, but Bob can. ${ }^{54}$ The respective tokens are stored in an account in the user's wallet.

Tokenisation is the technical process for producing a token. It is also used in projects to tokenise goods, meaning that the goods are marked with an ID or tracking code, which is represented by a token on the blockchain. All other layers, such as transport, enforcement and provenance can then be associated with tokenised goods. Furthermore, rights holders may authorise other parties to create and handle tokens on their behalf, or record events and information for their rights or goods. ${ }^{55}$

There are basically two ways of creating a token on a blockchain: mining and minting. As described, the nodes of a blockchain network are rewarded in cryptocurrency, meaning with tokens that represent the respective cryptocurrency, for mining the next block. These tokens are also referred to as (native) coins. ${ }^{56}$ The minting of tokens, on the other hand, is performed by smart contracts ${ }^{57}$. A smart contract function is called that can create an unlimited number of tokens. There are various standards to facilitate the process. Smart contract standards are, e.g., Ethereum's ERC-20, ERC-721, ERC-1155 or ERC-725..$^{5}$

However, there are different kinds of tokens, which can be generally distinguished by two characteristics, their purpose and their fungibility:

Based on the purpose, i.e., the intended use, relevant tokens in the IP ecosystem are - although there are more (suband hybrid) types - categorized in currency (coin or payment), security, and utility tokens. Currency tokens are assigned a value to be used as cryptocurrency, i.e., as a means of payment, such as Bitcoin or Ether. ${ }^{59}$ These currency tokens are also used to pay transaction fees-also called gas fees, e.g. for deploying a smart contract or sending token X from Alice to Bob. Security tokens represent securities, i.e., they serve as financial instrument and therefore subject to special regulatory oversight. ${ }^{60}$ Although in some cases the security character of an IP-related token is discussed, most relevant for IP matters are utility tokens. Utility tokens can be defined as a type of crypto-asset intended to provide digital access to a good or service, available on DLT, that is only accepted by the issuer of that token ${ }^{61}$. They can represent, for example, any IP right or IP-protected good, or provide access to an event or any other IP asset. Examples are tokens that give access to music on a platform or any digital or physical art or fashion product. Nonetheless, the utility token category is very diverse and such tokens might possess more than one utility attribute ${ }^{62}$. The differentiation between the different purposes of a token is important to assess whether certain regulations apply. In Germany, for example, the German Banking Act (Kreditwesengesetz, KWG) ${ }^{63}$ may apply to all types of tokens that are crypto-assets as defined by the KWG, while in the EU, the Markets in Crypto-Assets Regulation (MICAR) - that possibly will be aplicable already next year - addresses utility tokens, for example, but not tokens that are securities as defined by Markets in Financial Instruments (MiFID II) - Directive 2014/65/EU.

54 In detail, Markus Kaulartz/Robin Matzke (2018). Die Tokenisierung des Rechts, NJW, 3278.

55 European Commission (EC) and EUIPO (2019). Anti-Counterfeiting Blockchain Use Case <https://euipo.europa.eu/ohimportal/en/ web/observatory/blockathon> accessed on July 20, 2021.

56 Kaulartz/Matzke, Die Tokenisierung des Rechts, NJW 2018, 3278.

57 A further possibility currently practiced with NFTs is a "soft minting", i.e., initially the token is not minted but instead the ownership is documented on the blockchain in a simpler and cheaper way and then the "owner" can decide when to press the "mint" button so that the NFT is "hard minted" and accessible to the NFT ecosystem, e.g., Etherscan.

$58<$ https://tokenmint.io/blog/mintable-erc20-token-explained.html>. The aforementioned ERC20 token is a fungible token, each one is equal to the other. Examples of non-fungible tokens include ERC721 tokens <https://docs.ethhub.io/guides/a-straightforwardguide-erc20-tokens/>. ERCs stand for Ethereum Request for Comments and basically defines a set of rules that can be approved as standard to implement tokens on the Ethereum blockchain <https://docs.ethhub.io/built-on-ethereum/erc-token-standards/whatare-erc-tokens/> accessed on July 20, 2021.

59 If the cryptocurrency is bound to a Fiat currency (e.g., a certain token has the value of $\$ 1$ ), the tokens are referred to as "stablecoins".

60 In the EU the Directive 2014/65/EU (MiFID II) <https://eur-lex.europa.eu/legal-content/EN/TXT/?uri=CELEX\%3A32014L0065> accessed on July 20, 2021; the new law on the introduction of electronic securities ("eWpG") by the German Government <https:/ /www.gesetze-im-internet.de/ewpg/index.html> accessed on August 1, 2021; in the USA Sec. 2(a)(1) of the US Security Act, <www.govinfo.gov/content/pkg/COMPS-1884/pdf/COMPS-1884.pdf> accessed on July 20, 2021;

61 Art. 3 1.(5) of the European Commission's proposal for a Regulation on Markets in Crypto-assets (MiCAR) <https://eur-lex.europa.eu/ legal-content/EN/TXT/?uri=CELEX\%3A52020PC0593> accessed on July 20, 2021; Julia Hugendubel, Impact of the proposed EU Regulation on Markets in Crypto-assets for IP applications (2021)<https://ipkitten.blogspot.com/2021/01/impact-of-proposedeu-regulation-on.html> accessed on July 20, 2021.

62 ITSA, International Token Classification (ITC), Version 1.0 (01 March 2021).

63 In detail, Bafin, Merkblatt, Zweites Hinweisschreiben zu Prospekt-und Erlaubnispflichten im Zusammenhang mit der Ausgabe sogenannter Krypto-Token (2019). 
Additionally, tokens are differentiated with regard to their changeability, i.e., fungible and non-fungible tokens. Fungibles are those tokens that can be substituted for the same kind of token, such as currency or gold. All fungible tokens or fractions of such tokens are equivalents. Non-fungible tokens, on the other hand, are one-of-a-kind and represent unique assets or goods. ${ }^{64}$

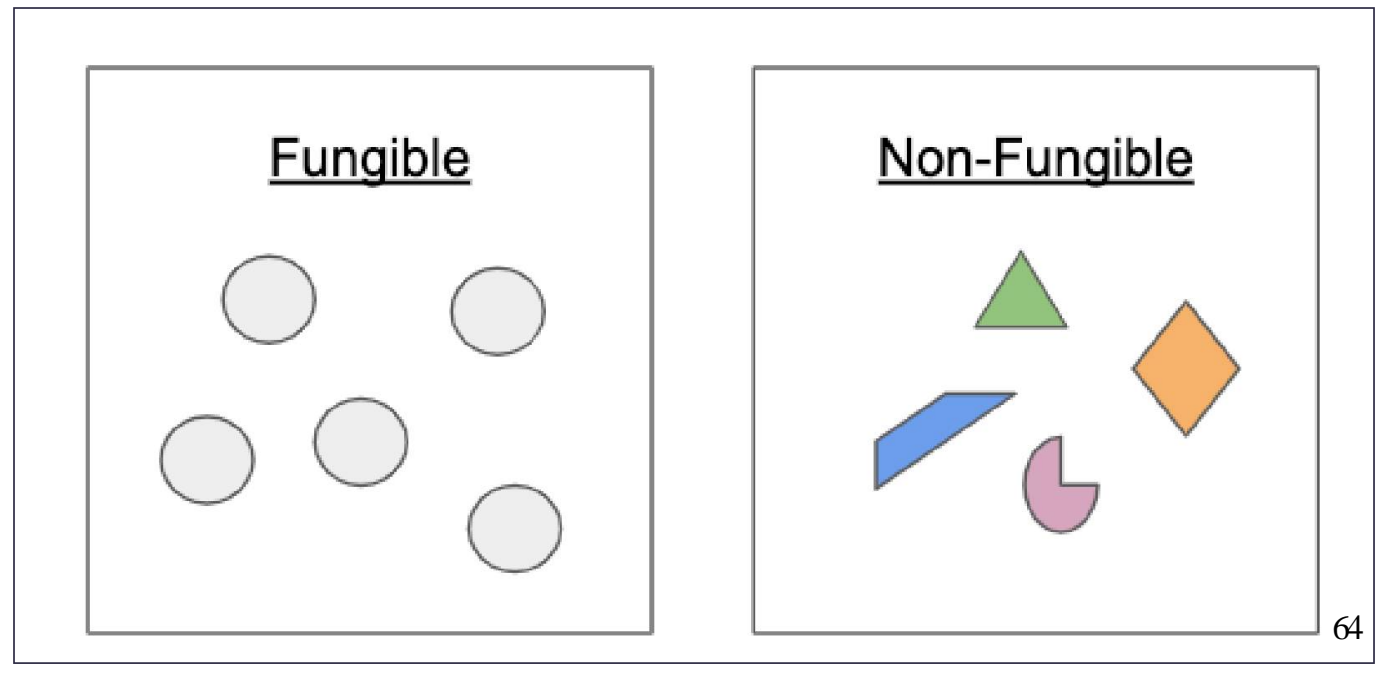

Fungible tokens can be issued to create access rights for an IP, for example to music or movies. Non-Fungible Tokens (NFTs) can represent art and, as is the case with the Cryptokitties ${ }^{65}$, serve as unique digital collection items.

Important for their legal evaluation is to see the possible separation between a token and the underlying asset linked to the token, e.g., a photography, a music piece or a movie. This is the consequence of the fact that tokens, in particular NFTs, only embody metadata - using a token Uniform Resource Identifier (URI) - that refers to the desired content outside of the smart contract. ${ }^{66}$

From a legal perspective, a common misunderstanding concerning tokens, specifically NFTs representing IPrights and goods, is their copyright protection. Copyright will generally not apply to a non-fungible token as such, if the NFT is minted using a common smart contract standard such as the ERC-721. Generally, only the copyright protection of the work linked to the NFT will be possible. Thus, unless specific regulations for crypto-assets apply-currently in most cases given the lack of such laws - the legal determination of ownership and the transactions of a token have to follow existing civil laws.

Users of a blockchain might also use a token in a legally binding way according to the civil code rules of a respective country. From a legal perspective, fungible tokens might be defined only by class ("Gattungsschuld", $§ 243$ German Civil Code (BGB)), whereas non-fungible tokens could be seen as unique assets ("Stückschuld") that can be transferred only in this specific form ${ }^{67}$. Nevertheless, this legal classification of tokens under current law is problematic, as only "things" are distinguished between classes and unique assets. Because things are only corporeal objects as defined by the BGB, tokens cannot be classified as things. ${ }^{68}$ Furthermore, a property right to NFTs according to $\S 903$ BGB $^{69}$ and an analogy to $\S \S 929$ et seq. BGB for the transfer of NFTs are discussed ${ }^{70}$.

64 <https://docs.ethhub.io/guides/a-straightforward-guide-erc20-tokens/> accessed on July 20, 2021.

65 <www.cryptokitties.co/> accessed on July 20, 2021.

66 The URI can be a URL using a JSON format that contains the description of the NFT, the link to a file, the image to be displayed in the user's wallet and the name of the NFT. To avoid any changes to the URI's content, it should not contain a traditional HTTP link as the content can be easily replaced, but a content identifier (CID) that is directly derived from the data itself and links to the original data (e.g. using InterPlanetary File System (IPFS), a protocol and peer-to-peer network for storing and sharing data in a distributed file system). A CID is a label used to point to specific information, e.g. a photography. The IPFS content identifier does not indicate where the content is stored. CIDs are based on the content's cryptographic hash. Thus, thus any difference in the content will produce a different CID and the same content using the same settings will always produce the same CID.

67 With consequences such as the exclusion of the duty of performance pursuant to $§ 275$ BGB ("Unmöglichkeit").

68 An exception is stipulated in the new law on the introduction of electronic securities ("eWpG"). The eWpG defines in $\S 2$ ( 3 ) that "an electronic security shall be deemed to be an object within the meaning of section 90 of the Civil Code", including crypto securities (see the definition for electronic securities in $\S 4 \mathrm{eWpG}$ ) <https://www.gesetze-im-internet.de/ewpg/index.html> accessed on August 1, 2021.

69 Markus Kaulartz, Alexander Schmid, Rechtliche Aspekte sogenannter Non-Fungible Tokens (NFTs), CB 2021, $298-302$.

70 Thomas Hoeren/Wolfgang Prinz (2021). Das Kunstwerk im Zeitalter der technischen Reproduzierbarkeit NFTs (Non-Fungible Tokens) in rechtlicher Hinsicht CR, 565-572. 
Some regard tokens as "other rights" ( $\$ 413 \mathrm{BGB}),{ }^{71}$ meaning a token might then be transferred as a claim pursuant to $\S \S 413,398 \mathrm{BGB}^{72}$. Moreover, tokens are considered as "another right" according to tort law provisions ${ }^{73}$. Furthermore, other classifications such as "Ownership of documents of debt" according to Section 952 BGB are discussed ${ }^{74}$.

\section{Use Cases of Blockchain Technology in the IP Ecosystem}

Aside from the traditional IP office matters to protect blockchain technology there is extensive research on specific blockchain applications in the IP ecosystem. ${ }^{75}$ Various industries have started blockchain projects for IP protection, such as, IPwe in the private sector or IP offices such as EUIPO and IP Australia. The WIPO states that IP offices all over the world are studying blockchain technology. ${ }^{76}$

Discussed applications for IP include evidence of creatorship or ownership, digital rights management; establishing and enforcing IP agreements, such as licensing or purchasing; combating counterfeiting ${ }^{77}$; IP office processes; providing evidence of genuine and/or first use in trade and/or commerce. ${ }^{78}$ The following chapters will give an overview about applications of distributed ledger technology, in particular blockchain, and why and how it is used in the IP ecosystem. The focus will be on the use of DLT for evidence of creatorship or ownership, licensing of IP rights, combating counterfeiting and its use by IP offices.

\subsection{Evidence of Creatorship or Ownership}

According to the European Commission blockchain could be a "significant advantage for the right holder" to secure intellectual property such as copyrights and trademarks, but also any ideas, concepts or trade secrets, "especially in the Chinese context where blockchain-based evidence is recognised in court." "A9 Additional applications include unregistered designs and the disclosure of IP to the public. ${ }^{80}$

The data of any IP on a blockchain, possibly with further information such as the creator's identity, makes it possible to generate a timestamped record ${ }^{81}$ with much higher credibility then any screenshot of a website or paraphrasing of a document can provide. Moreover, it is suggested to use blockchain for IP assets during their development phase as proof of ownership. Because IP or other information can be represented as a cryptographic hash on a blockchain, no third party will know about the deployed asset. However, in case of a later dispute the information on the blockchain can prove that the development was at a certain time in the possession of the person or legal entity.

It is worth noting that WIPO had introduced an off-chain tokenisation tool for any kind of content represented in a digital file_-such as IP rights. Since May 2020, the organization offered a platform allowing users to create a cryptographic token by scanning any information such as ideas, trade secrets or IP with a hash function. Nevertheless, if the hash from the original file and the hash of the token match, the WIPO token is validated. If the original file is modified in any way, the comparison will not succeed. The same holds true for any change to the WIPO token. The purpose of this so-called

71 As far as it is a non-fungible token that represents goods (e.g. IP protected products) the application of property law principles i.e., the consideration of token as a "thing" - could lead to a legal synchronization of the individual token and the good that is represented by the token. On the other hand, in cases where the token is related to a right such as to access music or movies or even events (i.e., rights) from a legal perspective, the classification as a "right" would be more adequate. To the extent that transactions of off-chain assets follow their common legal provisions, however, the representing token on-chain could follow as an annex right.

72 In detail, Kaulartz/Matzke, Die Tokenisierung des Rechts, NJW 2018, 3280. Concerning security transactions and the German Security Trading Act, BaFin <https://bit.ly/2TXROTj> accessed 20 July 2021.

73 In concreto $\$ 823$ (1) BGB, Stefan Möllenkamp/Leonid Shmatenko, in: Hoeren/Sieber/Holznagel, MMR-HdB, Part 13.6, Blockchain und Kryptowährungen, para. 34.

74 In detail, Philipp Maume/Lena Maute/Mathias Fromberger, Rechtshandbuch Kryptowerte (2020), Kapitel 2 Kryptotoken im Rechtsverkehr, § 4 Die Rechtsnatur von Kryptotoken, para. 54.

75 WIPO Report on Task No. 59 of the Committee on WIPO Standards, CWS/7/6 (2019) <www.wipo.int/meetings/en/ details.jsp?meeting_id=50414> accessed on July 20, 2021.

76 WIPO PP "Blockchain Whitepaper Project" (2020) 17 <www.wipo.int/meetings/en/details.jsp?meeting_id=57568> accessed 20 July 2021

77 Julia Hugendubel and Adrian Zarm (2021). Blockchain-Anwendungen als Anti-Counterfeit-Maßnahme, IPRB 2021, $42-47$.

78 Birgit Clark (2019). Crypto-Pie in the Sky? <https://stanford-jblp.pubpub.org/pub/blockchain-and-ip-law/release/1> accessed on August 7, 2020; WIPO PP "Blockchain Whitepaper Project" (2020) 19 <www.wipo.int/meetings/en/details.jsp?meeting_id=57568> accessed on July 20, 2021.

79 European Commission Guide (2019). IPR Protection for AI Technology \& Application of Blockchain in China <https://bit.ly/ 3 mihxBX> accessed on July 20, 2021.

80 See <https://wipoproof.wipo.int/wdts/use-cases.xhtml> accessed on July 20, 2021.

${ }^{81}$ Clark (2019). Crypto-Pie in the Sky? <https://stanford-jblp.pubpub.org/pub/blockchain-and-ip-law/release/1> accessed on August 7, 2020; Clark (2018). Blockchain and IP Law: A Match made in Crypto Heaven? <www.wipo.int/wipo_magazine/en/2018/01/ article_0005.html> accessed on July 20, 2021. 
"WIPO PROOF" was to provide a tool to prove the prior existence of a specific work. Nevertheless, WIPO now announced that the service will be formally discontinued on February 1, 2022. ${ }^{82}$

A token on blockchain, on the other hand, could simplify its use with further transactions, for example licensing or purchasing through smart contracts. Blockchain technology allows IP usage, such as streaming a song, to be granted automatically and without intermediaries for anyone that fulfils the pre-programmed conditions.

Nonetheless, it should be noted that merely deploying IP content on a blockchain generally only serves as an indication of creatorship. The tokens are limited to proving that the account owner was in possession of the tokenised asset at a given time, which is basically a kind of prima facie evidence of creatorship or ownership of IP. ${ }^{83}$ Tokens might also be regarded as a presumption of creatorship or ownership (Art. 15 Berne Convention; $§ 10$ German Copyright Act).

Against this backdrop, the use of blockchain technology seems to provide added value as it is used beyond the mere timestamped possession record of an idea/a work, such as for licensing to third parties. For one-off documentation - i.e., merely for prima facie evidence of creatorship or ownership - the use of a simple token such as the WIPO PROOF might be an easier yet secure solution (depending on the compared blockchain application ${ }^{84}$ ). Although it is likely that hashbased information from blockchains will sooner or later be permissible in courts all over the world, uncertainty about its legal acceptability currently remains. On the other hand, the argument against the WIPO PROOF could be that using a blockchain opens up the possibility of not being dependent on the availability of a tool from a particular organization, and that blockchain applications can also provide more cost-effective solutions.

A new use case could be the blockchain-based copyright register currently under discussion (in Germany with regard to $\S 66$ and $\S 138$ Copyright Act). ${ }^{85}$ Countries such as the USA and Brazil already have a copyright register, although its use can only have a declaratory character according to Art. 5 (2) of the Berne Convention. Despite the fact that a copyright exists from the moment a work is "fixed" rather than requiring registration, a blockchain-based copyright register could lead to more transparency, especially with regard to chains of rights. Such a blockchain-based registration is also discussed in the EU Commission's IP Action Plan ${ }^{86}$.

\subsection{Licensing and Purchasing}

IP licensing is estimated to be one of the most relevant use cases to benefit from blockchain technology. ${ }^{87}$ DLT, with its self-executing smart contracts, is considered an economically viable instrument especially for frequent, standardised transactions such as the licensing or purchase of music, movies, photography, or computer games. Furthermore, it could be used in the case of franchising businesses, which typically involve a standard transfer of IP rights. A blockchain solution could increase transparency, reduce administration and costs, and accelerate processes. It is seen as an opportunity for IPright holders to monitor and track the distribution of registered as well as unregistered $\mathrm{IP}^{88}$. According to the Commission's IP Action Plan, the use of high-quality metadata and technologies such as blockchain can also help to "achieve greater transparency and better rights data management, particularly in the area of copyright and in terms of better identification of rights holders." 89

An early use of blockchain technology to distribute IP rights came from UK music singer-songwriter Imogen Heap in 2015. She released her new single using a smart contract on the blockchain Ethereum; the song could be purchased with the cryptocurrency Ether. Revenue from sales was divided among Heap (91.2\%) and the other artists who worked with her (1.3\% each for six people).${ }^{90}$ After that, more and more blockchain-based platforms for copyright distribution emerged, such as Ujomusic ${ }^{91}$, Mycelia (founded by Imogen Heap), ${ }^{92}$ Soundac (formerly Muse) ${ }^{93}$ and Theta.tv ${ }^{94}$. Artists

82 In detail, <https://wipoproof.wipo.int/wdts/how-it-works.xhtml> accessed on July 20, 2021

83 See <https://wipoproof.wipo.int/wdts/use-cases.xhtml> accessed on July 20, 2021; European Commission Guide, IPR Protection for AI Technology \& Application of Blockchain in China <https://bit.ly/3mihxBX> accessed on July $20,2021$.

84 E.g. applications such as those offered by <https://originstamp.com/> accessed on July 22, 2021, offer blockchain-based timestamping in minutes.

85 In detail, Hohn-Hein/Barth (2018). Immaterialgüterrechte in der Welt von Blockchain und Smart Contract, GRUR, 1089 , 1092.

86 <https://eur-lex.europa.eu/legal-content/EN/TXT/HTML/?uri=CELEX:52020DC0760\&from=EN> accessed on July 20 , 2021.

87 WIPO Blockchain Survey (2020) <www.surveygizmo.com/s3/5651590/Blockchain-IP-Ecosystem-Survey> accessed on July 29, 2020 .

88 Emmanuelle Ganne (2018). op. cit.

89 <https://eur-lex.europa.eu/legal-content/EN/TXT/HTML/?uri=CELEX:52020DC0760\&from=EN> accessed on July 20 , 2021.

$90<$ <ww.finivi.com/music/> accessed on July 20, 2021.

<https://ujomusic.com/> accessed on July 20, 2021.

<http://myceliaformusic.org/> accessed on July 20, 2021.

<https://soundac.io/> accessed on July 20, 2021.

<www.thetatoken.org/> accessed on July 20, 2021. 
also started to distribute their creative work using non-fungible tokens, prominent examples being Pak and Signe Pierce. $^{95}$

Blockchain-based platforms manage IP rights through smart contracts and automatically send the artists their revenues after the user has licensed/purchased their work. The programmed smart contract generally contains as a condition the retrieval of the license/purchase item ("if") and calculates the respective royalties for this ("then"). The royalties are automatically debited from the consumer's account. Additionally, the right of use can be programmed for a limited amount of time with an option to renew automatically or manually. Moreover, a smart contract can monitor the time and number of requests made by the licensee or the granting of sublicences. Even micro-licensing of small fractions of an IP work is discussed ${ }^{96}$. Corresponding licence chains can then be fully tracked via the respective blockchain.

In the future it is very likely that standard licence agreements will be administrated using smart contracts. This will help to automatize transactions, thus reduce time and costs. At the same time, the efficiency of IP rights management can be increased by connecting and updating the data automatically according to the entries in the individual IP office's registries and pursuant to the licensing in the relevant area where the IP is protected. Combined with artificial intelligence, such as searches for IP violations online, IP protection and the administration of IP rights will be simplified. Especially concerning large IP portfolios, the follow-up costs, such as for administrating continuous payments to IP offices or to validate the status quo of an IP right for a due diligence report, will be significantly reducible.

Against this background, collecting societies such as GEMA in Germany, SIAE in Italy or DAMA in Spain eventually might have to adapt their work concept.

Beyond the licensing and distribution of digital IP on blockchain platforms, applications for physical IP-protected goods such as fashion are being discussed. Mirroring the distribution of non-digital IP-protected goods on-chain is nevertheless a challenge. Suggestions to guarantee that the off-chain ownership status matches the on-chain status include creating virtual twins and linking the off-chain data to the chain by using QR codes, NFC or RFID tags. ${ }^{97}$

In addition to the main smart contract conditions, smart contract dispute resolutions are developed to resolve potential disagreements. ${ }^{98}$ The idea is for the parties to agree in advance on the respective arbitration rules that will apply in the event of a dispute. A smart contract could accordingly be programmed both to execute the main services and to include—possibly self-executing—rules to resolve potential disputes.

To overcome the niche market of the present offers, the challenge is to develop decentralised applications (DApps) that are attractive to users, while at the same time guaranteeing the privacy of users and complying with legal requirements. Developments such as the buying and selling of certain cryptocurrencies via credit card or Paypal wallet ${ }^{99}$ might accelerate this process.

Legal challenges for license agreements executed automatically with smart contracts are, e.g., the implementation of consumer protection provisions such as the mandatory instructions on withdrawal in the $\mathrm{EU}^{100}$.

\subsection{Anti-Counterfeiting}

According to the EUIPO 2020 Status Report on IP right infringement, the volume of counterfeiting in trade could be about $6.8 \%$ of total EU imports, or $€ 121 \mathrm{bn}$, while the direct economic cost of counterfeiting in the EU market is estimated at $€ 50$ bn per year in lost sales. ${ }^{101}$ Only the amount of counterfeit content on Instagram grew $341 \%$ between 2016 and 2019102. Counterfeit products are more than just a nuisance to all IP owners. Conventional identification tools such as barcode systems, serial numbers, UPC codes or paper certificates seem to offer only insufficient protection.

\footnotetext{
<https://a16z.com/2020/11/22/crypto-for-creators-tokenized-collectibles-nfts/> accessed on July 20, 2021.

$<$ https://bit.ly/2GBALDE> accessed on July 20, 2021.

97 <https://euipo.europa.eu/tunnel-web/secure/webdav/guest/document_library/observatory/documents/reports/ 2021_Anti_Counterfeiting_Technology_Guide/2021_Anti_Counterfeiting_Technology_Guide_en.pdf > accessed on July $29,2021$. In detail, see chapter II 3.

98 In detail, Kaulartz (2018). op. cit., para. 19, 20; Thomas Legler, Arbitration of Intellectual Property Disputes, 37 ASA Bulletin, $2 /$ 2019 289, (303) <https://bit.ly/3m9DNhP>; e.g. "CodeLegit" from Datarella <https://codelegit.com/tag/arbitration-library/> accessed on September 15, 2020.

99 <https://www.bbc.com/news/technology-54630283> accessed on July 20, 2021.

100 Additionally, with the EU Commission`s Proposal on Markets in Crypto-assets Regulation (MICAR), expected to enter into force in 2022, "Issuers of crypto-assets, other than asset-referenced tokens and e-money tokens, shall offer a right of withdrawal to any consumer who buys such crypto-assets directly from the issuer or from a crypto-asset service provider placing crypto-assets on behalf of that issuer." Article 12 (1) MICAR.

$101<$ <ttps://euipo.europa.eu/ohimportal/en/web/observatory/status-reports-on-ip-infringement> accessed on October 10, 2020.

$102<$ file:///C:/Users/Jules/Documents/Studium/FGV/TCC/2021_Monitoring_and_analysing_social_media_in_relation_to_IPR_ Infringement_Report_FullR_en.pdf> accessed on April 22, 2021.
} 
This could explain the enormous interest of IP offices and private companies that seek to combat counterfeiting of IP-protected goods with blockchain initiatives. Prominent examples are the EUIPO and the Australian IP office anticounterfeiting initiatives, as well as those of luxury brand owners such as Louis Vuitton, Moncler or Salvatorre Ferragamo. The declared purposes of using DLT for products include anti-counterfeiting infrastructure, safeguarding intellectual property, tracking supply chains, alerting rights holders and assuring authenticity for authorities as well as end users. ${ }^{103}$

\section{a. Technical Concepts to Combat Counterfeits with Blockchain}

As early as 1996, Nick Szabo had already predicted that "[s]mart property might be created by embedding smart contracts in physical objects" 104 . The colored coins Whitepaper in 2012 then combined this idea of smart property with blockchain. ${ }^{105}$ Current anti-counterfeiting projects have pursued this idea by seeking to track products or any physical asset using QR codes, RFID tags or other tracking tools such as crypto anchors ${ }^{106}$ to record the off-chain facts onchain. ${ }^{107}$ Therefore, a digital asset—often in the form of a unique token or another identifier — is created to represent the respective physical asset on a blockchain. A blockchain token in this case can simply embody data of the physical asset, such as its transactions, or it can be designed as an identical copy—called a virtual twin —of the information of the physical good.

This makes it possible to transfer the associated tokens on the blockchain as a transaction together with the offchain goods to provide transparent proof of their authenticity. The token on the specific blockchain should always reflect the current ownership of the individual product. The chain of previous owners thus serves as proof of the product's authenticity. Off-chain facts are linked to the blockchain by scanning the respective code, among other things. Off-chain data is sent to the blockchain through so-called "oracles". An oracle is a proxy that transfers off-chain data to a smart contract that is then compiled and deployed on the blockchain. ${ }^{108}$ This way, potentially any details of a product transfer can be recorded with a timestamp and reflected in the status on-chain. ${ }^{109}$

An example is designer goods with an embedded chip or code that is linked by a smart contract ("if ... then") to a token on a blockchain. By scanning the code-e.g. with a smartphone-information such as authenticity, material origin, designer, trade route could be visible.

Because of the characteristics of DLT, the aforementioned tracking is also considered an enormous opportunity for customs officials who have to track down counterfeit goods. Since, according to the Agreement on Trade-Related Aspects of Intellectual Property Rights (TRIPS), WTO members can enable customs officials to confiscate goods infringing IP rights, the main obstacle for tracking counterfeits may not be a lack of competence, but the difficulty in identifying counterfeits as such. ${ }^{110}$

In addition, it is suggested to deploy import and export licenses on a blockchain and to make the permit accessible to customs authorities — including by using a QR code that can be scanned via smartphone-to verify its authenticity and validity. It is estimated that fake permits can be eliminated in this way. Additionally, smart contracts could be programmed according to the respective regulations to pay customs duties with the help of an oracle ("when the products arrive at the customs terminal, you pay duties in the amount of XY"). Interoperability of blockchain platforms with eCertify and essCert, a tool for chambers, exporters and freight forwarders to eliminate paper processing (Certificates of Origin), is also being considered ${ }^{111}$. Nonetheless, it should be noted that the chamber of commerce does not certify the true origin of goods, but only the exporter's statement. Using a DLT would not change this. ${ }^{112}$

\footnotetext{
103 EC and EUIPO, Blockathon Report <https://euipo.europa.eu/ohimportal/en/web/observatory/blockathon> accessed on July 20 , 2021; <https://les-ch.ch/the-need-for-distributed-ledger-technologies-in-the-fight-against-counterfeits/> accessed on July $20,2021$.

104 Szabo Smart Contracts: Building Blocks for Digital Markets (1996). <www.fon.hum.uva.nl/rob/Courses/InformationInSpeech/ CDROM/Literature/LOTwinterschool2006/szabo.best.vwh.net/smart_contracts_2.html>.

105 Meni Rosenfeld (2012). Overview of Colored Coins <www.google $\cdot \operatorname{com} / \mathrm{url}$ ? $\mathrm{sa}=\mathrm{t} \& \mathrm{rct}=\mathrm{j} \& \mathrm{q}=\&$ esrc $=\mathrm{s} \& \operatorname{source}=\mathrm{web} \& \mathrm{~cd}=$ \&ved=2ahUKEwiSou-v5qjwAhX7gf0HHRyrDUEQFjAAegQI AhAD\&url=http\%3A\%2F\%2Fwww.bitcoinspotter.nl\%2Fsites\% 2Fdefault\%2 Ffiles\%2FBitcoinX.pdf\&usg=AOvVaw2DVvvTSLiZ1q-ZSayySvkP> accessed 23 March 2021. Electronic copy available at: https://ssrn.com/abstract=3917801

106 <https://ieeexplore.ieee.org/document/8645638> accessed on July 20, 2021.

107 WIPO Blockchain Survey, op. cit., July 29, 2020.

108 In detail, chapter IV. 3. c)

109 EC and EUIPO (2019). Anti-Counterfeiting Blockchain Use Case <https://euipo.europa.eu/ohimportal/en/web/observatory/ blockathon> accessed on July 20, 2021

110 Emmanuelle Ganne (2018). op. cit.

$111<$ <ww.ecertify.com/why_ecertify/>, <www.esscert.com/> accessed 20 July 2021.

112 In detail, Emmanuelle Ganne, op. cit.
} 
Yet, to use blockchain as a reliable tool also for customs officials, standards that allow verification of the product's authenticity are indispensable. Otherwise, the counterfeiting manufacturer could very likely also forge a blockchain entry. This is why the suggested approach that a brand owner should inform customs officials about the individual security features of its genuine products in order to identify counterfeits ${ }^{113}$ could be difficult to implement. Additionally, an individual approach by each company would require customs officials to adapt the specific verification method chosen.

One solution could be to use an IP office or another authority to authenticate the issuing of the token that represents the individual physical asset. Obviously, this could not be achieved through individual product verification. One approach could be that the identity of a company is validated once and then the requests for authentication of a token issued by this recognised business are executed - automatically by a smart contract—by the responsible authority. In other words, if the company fulfils certain requirements, the authorities would append a trusted timestamp to the hash of the token and create a new hash signed with the authorities' private key. These qualified tokens could then be used for product verification.

Because of these somewhat complex approaches, there is criticism that common platforms and identification tools can offer simpler but equally effective anti-counterfeiting solutions. Critics even argue that it is not possible to prevent the copying of IP work or its sale without updating the ledger, where the on-chain token is simply the avatar of the off-chain good. ${ }^{114}$

\section{b. Examples of Blockchain Based Anti-Counterfeiting Projects}

In particular, EUIPO and the Australian IP office have launched anti-counterfeiting initiatives in cooperation with the private sector. Supporting partners of the EUIPO anti-counterfeiting blockchain project, which is still in its creation stage, include Adidas, Amazon, BMW Group and Chanel. The EUIPO's suggestion for rights holders is a "Blockchain Access Portal" of an "anti-counterfeiting blockchain" where they can create tokenised goods for their product lines. The portal will also allow rights holders to register delegated, trusted third parties to create tokens on their behalf and to access associated services. ${ }^{115}$ The EUIPO combating counterfeit project is also explicitly supported by the EU Commission as stated in its recently published IP Action Plan. ${ }^{116}$

The EU Enforcement Database (EDB) ${ }^{117}$ already contains information on products with granted IP rights, such as registered trademarks or designs, and is accessible by police and customs officials from the EU member states to identify counterfeits. The EUIPO considers interoperability with the EDB and its features of authorisation and product line definition as important for a blockchain solution. At the same time, the office assures that the EDB's confidential data is not stored in the blockchain. Moreover, only rights holders can allow the recording of goods, access to associated data as well as services on the blockchain. ${ }^{118}$

According to the EUIPO concept, a physical product will only be accepted by the next operator as a unique product identity in combination with its virtual twin in order to ensure the accuracy of the data on the blockchain. A delivery without the virtual twin will raise a "red flag" in a reporting system to identify counterfeits. In addition, transport or shipment details will be recorded on the blockchain to verify the authenticity of the contained goods. The monitoring of events in the supply chain and a risk warning system with notifications to right holders and enforcement authorities are also being discussed. ${ }^{119}$

The Australian IP office has already started trialing an anti-counterfeiting initiative called "Smart Trade Mark" with industry and government partners. The government initiative seeks to enable brands to better protect themselves against misuse and counterfeiting by deploying the data on a blockchain, although it remains unclear which data will be anchored there. Owners or agents of a trademark are allowed to exclusively request a Smart Trade Mark linking their authentic products or services to their official trademark. To this end, the Smart Trade Mark platform digitally links trademark owners to the government register to prove their authentic ownership of a brand. With a "trust badge", trademark owners should be able to control the use of their trademarks. The idea is that the trust badge provides visual

113 Birgit Clark (2018). Blockchain and IP Law: A Match made in Crypto Heaven? <www.wipo.int/wipo_magazine/en/2018/01/ article_0005.html> accessed on July 20, 2021.

114 Michèle Finck/Valentina Moscon (2019). Copyright Law on Blockchains: Between New Forms of Rights Administration and Digital Rights Management 2.0, IIC, 77, 98.

115 <https://euipo.europa.eu/ohimportal/en/web/observatory/blockathon> accessed on July 20, 2021.

116 < IMMC.COM\%282020\%29760\%20final.ENG.xhtml.2_EN_ACT_part1_v10.docx (europa.eu)> accessed on July 20 , 2021.

17 <https://euipo.europa.eu/ohimportal/en/web/observatory/enforcement-database1?inheritRedirect=true> accessed 20 July 2021.

118 EC and EUIPO, Anti-Counterfeiting Blockchain Use Case (2019)<https://euipo.europa.eu/ohimportal/en/web/observatory/ blockathon> accessed on July 20, 2021.

$119<$ https://bit.ly/3ooOQFu> accessed on July 20, 2021. 
proof of a brand's authenticity, as this badge displays details that are also visible to consumers, such as the places where they are sold and their supply chains. ${ }^{120}$

Private companies have also started to use blockchain in an effort to combat counterfeit products and boost brand confidence. While companies like Moncler and Salvatorre Ferragamo use existing blockchains such as Ethereum, which collect data through RFID chips and QR codes that are sewn into their fabrics, embedded into shoe soles and other fashion items to verify their authenticity, other brands have developed their own DLT. A prominent example is "AURA" from LVMH, which controls more than 60 prestigious luxury brands including Louis Vuitton, Dior and Rimowa, aiming to authenticate their products. ${ }^{121}$

Additionally, companies such as Datarella, Everledger or Lukso are focused on providing blockchain platforms and applications specifically for lifestyle industry and valuable goods. ${ }^{122}$

Particularly for high value goods, companies might also consider blockchain-based traceability in the second hand market. Nevertheless, despite the attractiveness of these tracing tools for anti-counterfeiting purposes, such applications must be carefully designed to comply with data protection and antitrust laws.

\subsection{IP Registration and Administration}

The worldwide registration of IP is increasing rapidly. The Patent Cooperation Treaty (PCT) shows an increase of $71 \%$ between 2009 and 2019, for the Madrid System (trademarks) about $78 \%$ and for the Hague System (industrial designs) an impressive growth of $167 \% .{ }^{123}$ The extent to which the use of blockchain technology could increase the efficiency of IP office processes to handle this huge rise in IP registrations is discussed below.

\section{a. Smart IP Offices}

The WIPO itself aims to guide IP offices and Organizations "to manage, store, process, exchange and disseminate IP data using Blockchain". The organization is even considering a blockchain-based "unique and global IP registry". The announced WIPO standards will support a harmonised blockchain development and interoperability among IP offices. ${ }^{124}$

This development of using DLT as an instrument of administration as well as decision-making is already established in various countries all over the world. In Switzerland, blockchain is used for tax payments, e-voting and identity management; Estonia and Sweden have blockchain-based government and healthcare projects; in Cyprus, Malta and France, there are blockchain projects for education and certification; Sweden, UK and Ukraine launched initiatives for title and asset registration with blockchain; ${ }^{125}$ In China, the first internet courts have been established since 2017, interacting with Artificial Intelligence (AI) as well as blockchain and therefore called "Smart Courts". ${ }^{126}$ A next step might be that IP offices, as they adapt more and more to AI-based solutions ${ }^{127}$, eventually in combination with DLT, gradually become "Smart IP offices".

While current national laws require the approval of IP offices to achieve a registered IP, only consortium blockchain models might serve as a possible solution for IP office processes. ${ }^{128}$ IP offices controlled by a consortium blockchain would allow access control. This regulation would not be feasible with a mere public blockchain. Although overcoming the requirement of a trust-building central authority is considered to be one of the main advantages of blockchain, it contradicts current government regulations to substitute its authentication function ${ }^{129}$. A private one, on the other hand, would not be accessible to everyone.

The EUIPO launched such a consortium blockchain for the data of TMView and DesignView, using a Hyperledger protocol of the Linux Foundation with Malta as first country joining the network. In July, the Industrial Property

$20<$ https://smarttrademark.search.ipaustralia.gov.au/\#product-trial> accessed on July 20, 2021.

$121<$ https://auraluxuryblockchain.com/> accessed on June 1, 2021.

122 <https://datarella.com/track-trust/>, <www.everledger.io/industry-solutions/luxury-goods/>, <www.lukso.network/> accessed on July $20,2021$.

123 <www.wipo.int/pressroom/en/articles/2020/article_0005.html> accessed on July 20, 2021.

124 WIPO Report of the Committee on WIPO Standards CWS/7/6 (2019) <www.wipo.int/meetings/en/details.jsp? meeting_id=50414> accessed 20 July 2021; WIPO Blockchain Survey, question no. 51 op. cit., accessed on July 29, 2020.

125 <https://stanford-jblp.pubpub.org/pub/blockchain-evidence-courts-china/release/1> accessed on July $20,2021$.

126 Mimi Zou, 'Smart Courts' in China and the Future of Personal Injury Litigation (March 11, 2020). Journal of Personal Injury Law (forthcoming, June 2020), <https://ssrn.com/abstract=3552895> accessed 20 July 2021.

127 <www.wipo.int/about-ip/en/artificial_intelligence/policy.html> accessed 20 July 2021.

128 In 2019, Fabian Vogelsteller, LUKSO Founder and Ethereum developer, strongly recommended that the WIPO invest in developers and build up a consortium blockchain for all its members; Henry Cuschieri from ISO agreed: "I think it is totally over due." <https:/ /c.connectedviews.com/05/SitePlayer/wipo?session=104396> accessed 20 July 2021.

129 Hohn-Hein/Barth, Immaterialgüterrechte in der Welt von Blockchain und Smart Contract, GRUR 2018, 1089 , 1092. 
Registrations Directorate (IPRD) of the Maltese Commerce Department installed an IP Register node and transferred 60,000 records to TMView and DesignView through the EUIPO blockchain network. ${ }^{130}$

Furthermore, the International Chamber of Commerce (ICC) together with the Singapore Government has already launched a consortium blockchain network for enterprises (called "TradeTrust"), i.e., a blockchain with a public interface for (private) companies. ${ }^{131}$ In its rough form, TradeTrust could be seen as a model for an IP office blockchain. It has a public interface, meaning that - transformed for IP offices-everyone could read the data such as the individual IP registrations. The features for IP holders and IP offices, however, would only be accessible with a specific (blockchain) account. The IP office's blockchain(s) would distinguish between the specific information and its accessibility, as is already practiced, for example, on the EUIPO website. Data could be exchanged directly between IP offices and other authorities or between IP holders. Processes could be automated by smart contracts, and standards could be introduced to create smart contracts for IP holders to interact with licensees, among others.

A blockchain-based IP office system, used for example by all members of the Madrid Protocol, instigated or initiated by the WIPO, could probably achieve the highest efficiency in processing and managing IP, and would give unrestricted freedom regarding the feature set, such as selecting the most suitable consensus protocol. On the other hand, it is possible that IP offices and private distributors will use different blockchain applications, although this would be the most inefficient blockchain solution (participants might have to register for different applications, use different cryptocurrencies, and eventually undergo new authentication procedures for each platform). It is likely that in the beginning, there will be more than one solution with the participation of a limited number of IP offices, and then participation will eventually grow. Thus, the main challenge will be the interoperability between different applications. The more the IP offices unify and eventually use one platform or guarantee the interoperability of their applications, the more efficiency and effective IP protection could be achieved.

However, it is questionable why blockchain would be more effective for IP offices than harmonising procedures by using conventional technologies and online platforms. Current international developments such as the Digital Access Service (DAS) - a system that promotes the provision of priority and similar documents to participating offices without the need to receive and send multiple certified paper copies of an application ${ }^{132}$ _(joined by the EUIPO in 2020), ${ }^{133}$ already seem to provide high data availability. Furthermore, for the registration and administration procedures with IP offices in general, there is no trust problem that could be solved using blockchain technology. IP office registers enjoy a high level of credibility and are expected to be correct. Nor are there currently any intentions to replace IP offices as intermediaries, even if in the long term many office procedures could be performed by AI.

In this respect, the added value of using blockchain technology could derive from the automation of payment, administration and management of IP, possibly in combination with anti-counterfeiting tools, in a unified system. It is seen as potential to accelerate procedures such as control and approval processes for IP transactions with a digital authentication that can safely eliminate paper, avoid missing documents and ultimately be more cost effective. ${ }^{134}$ Higher security could also be achieved by decentralising and encrypting data and by controlling access and transactions with cryptographic keys. Timestamps, payments, and entries in the register-such as pursuant to Art. 111 European Union Trade Mark Regulation (EUTMR) — updates, renewals or cancellations could be carried out automatically by means of smart contracts. After the automatically processed application — which can already be pre-checked by means of e-filing to ensure it meets all the formal requirements — and its entry into a system that is linked to a blockchain, a smart contract could initiate the notification of the applicant about refusal or registration, depending on the IP office's decision or any pre-programmed conditions. A smart contract could then adjust the entries in the IP office's register accordingly. More steps would currently be required in the event of a partial refusal or opposition procedures. The numerous office fees such as under Annex 1 EUTMR could all be included, which would be due in case of underlying conditions such as the fee for the application for registration of a licence (Annex 1 EUTMR no. 26). Furthermore, depending on the features of the blockchain application, it could be connected with other use cases such as license agreements (Art. 26 EUTMR) and blockchain-based dispute resolutions.

\footnotetext{
30 https://euipo.europa.eu/ohimportal/en/web/guest/news/-/action/view/8793606> accessed on July $20,2021$.

<https://bit.ly/3etoJbZ> accessed on July 20, 2021.

<https://guidelines.euipo.europa.eu/1803372/1882144/designs-guidelines/2-3-3-wipo-das> accessed on July $20,2021$.

<www.tmdn.org/network/-/euipo-joins-the-wipo-digital-access-service-das-> accessed on July 20, 2021.

134 WIPO Blockchain Survey op. cit., accessed on July 29, 2020; Anne Rose (2020). Blockchain: Transforming the registration of IP rights and strengthening the protection of unregistered IP rights. <www.wipo.int/wipo_magazine_digital/en/2020/article_0002.html> accessed on July 20, 2021.
} 
Arguably, IP office processes could therefore be regarded as being predestined for the use of blockchain technology, not least as this could enable unified blockchain registers for all registered IP rights. Contrary to copyrights, where registration will not be mandatory (Art. 5 (2) of the Berne Convention), registration is constitutive in particular for trademark, patent and design besides the unregistered community design. Furthermore, these IP rights are already registered in online databases and "only" have to be used for respective blockchain applications.

Nonetheless, the claimed advantages depend on a vast development of blockchain applications for IP offices and their users. Additionally, the responsibilities of IP offices also depend on interpretation of law and facts as well as assessment of evidence; ergo they are difficult to pre-program. So far it is likely that blockchain-based solutions, even in combination with AI, will not be able to fulfil all IP office tasks in the near future. This is the case at least in as far as efficiency is not prioritised over accuracy, as pointed out by Zhang Wen, President of the Chinese Internet Court in Beijing, in relation to AI-based court decisions. ${ }^{135}$

\section{b. Smart IP Rights}

An example of a smart IP right is the aforementioned "Smart Trade Mark" blockchain-based anti-counterfeiting project by the Australian IP office. In addition, the first private entities built up blockchain based IP platforms, such as for patents by IPwe ${ }^{136}$ and for trademarks by Cognate ${ }^{137}$.

The WIPO task force sees high value opportunities in blockchain applications for processing IP rights, notably discussing "shared Trust Blockchain-based IP registries", including the issuing of recorded IP assets to reflect the chain of ownership for each registered IP in the register. Possible advantages are seen in data sharing among government agencies, which reduces data redundancy and ensures data integrity. Thus, the information shared by the WIPO indicates that it is not a question of whether, but only how blockchain technology will be integrated into IP office procedures. ${ }^{138}$ In particular, the following blockchain-based IP registrations are discussed:

It is suggested to simplify the registration process of a trademark by collecting information on the right preserving use of a trademark on a blockchain ledger, with the possibility to notify IP offices virtually about this use. The exact time and extent of use could be linked to the trade mark register. Thus, the register is also named "Smart Trade Mark Register", because it could reflect the state of the market as well as "the entire life cycle of a right" 139 . The WIPO task force as well as the EU Commission have already discussed IP right data sharing based on a "unified storage/repository of 3D models and images, using Blockchain technology" ${ }^{140}$. This could serve to document proof of use of a trademark pursuant to Art. 18 EUTMR, and provide evidence for the proof of the time of disclosure of a patent or design (Art. 54 European Patent Convention, Art. 5 Community Design Regulation). While proof of use is only required for trademarks, proof of priority or the act of publicity that initiates the grace period is relevant for industrial designs according to the Hague System and for patents pursuant to the PCT. With an exact timestamp, it could be verified whether a design or an innovation has to be considered new or not.

Smart contracts could also inform IP offices about a change of ownership or give an opt in function for registered trademark extensions, i.e., they could be extended and paid automatically by smart contract. This could mean lower administration costs, both for companies and for IP offices. Especially for large trademark portfolios in a vast number of countries, the transfer could be programmed with the value connected to the respective extension fee in the respective country.

\section{Opportunities for IP Applications}

WIPO`s opinion is that blockchain technology "has significant implications" for IP rights management. ${ }^{141}$ General opportunities of blockchain applications for IP matters are as follows:

$135<$ https://bit.ly/2JHINgw> accessed on July 20, 2021.

$136<$ https://ipwe.com/who-are-you> accessed on July 20, 2021.

$137<$ https://cognate.com/> accessed on July 20, 2021.

138 WIPO Report of the Committee on WIPO Standards CWS/7/6 (2019) <www.wipo.int/meetings/en/details.jsp? meeting_id=50414> accessed on July 20, 2021

139 Clark (2019). Crypto-Pie in the Sky? <https://stanford-jblp.pubpub.org/pub/blockchain-and-ip-law/release/1> accessed 7 August 2020 and Clark (2018). Blockchain and IP Law: A Match made in Crypto Heaven? <www.wipo.int/wipo_magazine/en/2018/01/ article_0005.html> accessed on July 20,2021.

140 WIPO Report of the Committee on WIPO Standards CWS/7/6 (2019) <www.wipo.int/meetings/en/details.jsp? meeting_id=50414> accessed on July 20, 2021; European Commission Guide, IPR Protec-tion for AI Technology \& Application of Blockchain in China <https://bit.ly/3mihxBX> accessed on July 20, 2021.

141 WIPO Blockchain Survey, op. cit., accessed on July 29, 2020. 


\subsection{Cost Reduction and Outsourced Data Storage}

Blockchain technology can reduce the need for intermediaries and therefore lower the costs of IP rights management. ${ }^{142}$ Depending on the chosen blockchain network, the need for central entities managing the transactions can even be eliminated. Blockchains, together with AI/IoT, could allow smart contracts to swiftly move assets, interact with physical objects and reduce mental and computational transaction costs ${ }^{143}$. It is seen as an option to program a smart contract so that it can be negotiated under certain conditions and linked to AI programs. ${ }^{144}$

Additionally, the use of DLT can reduce storage costs by outsourcing the storage of (encrypted) information to the network. The nodes' compensation for renting out their own hard drives could be made through micropayments. This way unused space could be used to further reduce the costs of file storage. Therefore, blockchain contracts could facilitate the development of a "decentralised file storage ecosystem". ${ }^{145}$

\subsection{Accelerated Procedures}

Furthermore, numerous transactions that take place on various platforms with different authorities—such as a platform for verification, for negotiations between the parties, for contacting the bank that transfers the money to another bank - can be unified by using blockchain technology. The result of the unification and automation process is a significant acceleration. ${ }^{146}$ The IP Helpdesk of the European Commission considers this a benefit with a drastic reduction in transactions and complexity. ${ }^{147}$ According to WIPO, adapting the DLT for supply chains will increase the efficiency, speed and volume of global trade, while at the same time reducing costs that are typically associated with international transactions. ${ }^{148}$ Acceleration potential is also seen in global collaboration between institutions, time savings such as in the issuing of certificates and transcripts and faster verification and background checks. ${ }^{149}$

\subsection{Potential Traceability of IP}

Blockchain technology theoretically enables recording of all IP assets and tracking of their status. The WIPO Blockchain Task Force even envisages a "digital reinvention of IP asset management". ${ }^{150}$ Basically, this calls for a "digital by default" principle as generally practised by the authorities in Estonia. ${ }^{151}$

\subsection{Micro-Licensing of IP Right}

Furthermore, blockchain technology allows transactions of IP right that can be remunerated with amounts of less than one cent. ${ }^{152}$ With regard to IP, the use of anything that can be partially accessed is being discussed, such as a fraction of a video or music piece ${ }^{153}$. As one Visa executive argued with a CEO of Coinbase on Twitter, this may promote transactions because generally no one would hesitate to spend a fraction of a cent. ${ }^{154}$

142 Ibid; Birgit Clark, Crypto-Pie in the Sky? (2019) <https://stanford-jblp.pubpub.org/pub/blockchain-and-ip-law/release/1> accessed on August 7, 2020.

143 Essentially, Szabo (1997). Smart Contracts: Formalizing and Securing Relationships on Public Networks. First Monday, 2(9) <https://bit.ly/3k5MGaq> accessed on July 20, 2021.

144 Bambara/Allen et al. (2019), op. cit., ch. 1, (29, 34).

145 Buterin (2013). op. cit., 11; G. Abinaya et al. (2019). Block Chain Based Decentralized Cloud Storage. International Journal of Engineering and Advanced Technology, 8(4) <www.ijeat.org/wp-content/uploads/papers/v8i4/D6206048419.pdf > accessed on July 20, 2021.

146 Clark (2019). op. cit.

$147<$ http://iprhelpdesk.eu/ip-highlights/ip-special-blockchain/blockchain-in-a-nutshell> accessed on July 20, 2021.

148 WIPO Blockchain Survey, op. cit., accessed July 29, 2020.

149 <www.industry.gov.au/data-and-publications/national-blockchain-roadmap/sectoral-opportunities> accessed on July 20, 2021.

150 WIPO Report on Task No. 59 of the Committee on WIPO Standards CWS/7/6 (2019) <www.wipo.int/meetings/en/ details.jsp?meeting_id=50414> accessed on July 20, 2021.

151 There, authorities use blockchain technology to follow a "once only principle" for any type of data submitted by a citizen, i.e., the same data should never be requested twice <https://e-estonia.com/press-review-a-digital-leadership-for-the-european-union/, https:/ /e-estonia.com/solutions/security-and-safety/> accessed on October 14, 2020. Nevertheless, an EU wide blockchain infrastructure (EBSI) is still under development. Additionally, a "Union-wide application of the 'once only' principle" is to be realised by the member states by 2023. Regulation (EU) 2018/1724, establishing a single digital gateway to access information, Regulation (EU) No 1024/2012. To what extent this will affect IP processes at the IPOs remains to be seen.

152 In detail, Hugendubel/Zarm, Blockchain-basiertes (Micro-)Licensing von IP-Rechten, CMS Update Gewerblicher Rechts-Schutz \& Kartellrecht 04/2021, <https://cms.law/de/deu/publication/blockchain-basiertes-micro-licensing-von-ip-rechten> accessed on July $29,2021$.

153 Thomas Euler (2019). The Tokenization Playbook for the Sports Industry <https://medium.com/liquiditeam/the-tokenizationplaybook-for-the-sports-industry-guide-blockchain-busines-87da13d33dfe> accessed on February 20, 2021.

154 <https://twitter.com/cuysheffield/status/1229029460403318786>; <https://bit.ly/3168Ryo> accessed on July $20,2021$. 


\subsection{Replacement of Paper Documentation and Insurance of Data Integrity}

Blockchain technology theoretically makes it possible to anchor all information which can be digitally stored in the ledger and make it potentially accessible to anyone from anywhere ${ }^{155}$. The access can be regulated depending on the blockchain protocol. The idea is that once a DLT has been chosen, the information does not have to be shared anymore $^{156}$. Therefore, it is assumed that paper might become dispensable if blockchain or other distributed ledger technologies are accepted as a reliable source of data ${ }^{157}$. However, new projects claim that this could be better accomplished by a distributed ledger network without using blockchain technology. ${ }^{158}$

\section{Challenges for IP Applications}

For the risk assessment of blockchain applications in the IP ecosystem, main challenges of blockchain technology for the IP ecosystem are presented below:

\subsection{Oracles and Possible Divergence of Token and the Rights or Goods Represented by the Token}

There is a challenge for every off-chain item that is to be represented on-chain. This is because there has to be a way to guarantee that the off-chain data matches the on-chain data. A blockchain itself cannot access data that is outside its network. The connection of off-chain data with on-chain data is made by so-called "oracles", presumably an analogy to the Matrix movie. Oracles are interfaces that provide a blockchain with external data. The data can come from hardware sensors or software. ${ }^{159}$ To use the external data on the blockchain oracles are linked to smart contracts and trigger an execution on the blockchain when the programmed conditions are met. ${ }^{160}$ Because oracles provide blockchain technology with information outside the chain, it can lead to false information on the chain if the data is incorrect or not updated. Difficulties in IP matters can be seen, such as in how to prove the existence or ownership of an IP right and how to synchronise facts off-chain with facts on-chain.

Current law does not provide for a general property right to data. On the other hand, various legal instruments already convey property like legal positions under given conditions and due to their fundamental technology neutrality. For personal data, the General Data Protection Regulation (GDPR) and, subsidiarily, the right of personality provide for individual claims that come close to the right of ownership. However, legal claims similar to ownership already exist today even for data without a personal reference. Under certain conditions, competition law also provides a certain degree of protection in addition to copyright law and other intellectual property law. Finally, property like positions in digital data can be assigned by contract. ${ }^{161}$

Also, the tokens that point to IP rights or protected goods still depend on the rights of the creator or owner and the rights attached to these tokens depend on the individual license or other agreements. Both the token issuer and the third party must consider the applicable laws to know their rights and obligations.

\subsection{Scalability Trilemma}

According to the scalability trilemma, blockchains try to have three properties, namely, scalability, decentralisation and security. Traditional blockchain techniques arguably enable only two of those three properties. ${ }^{162}$

While the blockchain community is growing enormously ${ }^{163}$, common scalability issues remain-not only but also-for the IP ecosystem. For instance, these issues are the volatility of cryptocurrency ${ }^{164}$ (e.g., for the payment to

155 Furthermore, a script is possible that gives access to specific features only if signed by different parties with their different private keys ("multi-validation"), Buterin (2013). op. cit., 12.

156 WIPO Report on Task No. 59 of the Committee on WIPO Standards CWS/7/6 (2019) <www.wipo.int/meetings/en/ details.jsp?meeting_id=50414> accessed on July 20, 2021.

157 Emmanuelle Ganne (2018). op. cit.

$158<$ https://hedera.com/> accessed on July 20, 2021.

159 <www.heise.de/ct/artikel/Blockchain-Technik-jenseits-von-Kryptogeld-4564147.html> accessed on July $20,2021$.

160 Bambara/Allen et al. (2019). AI, IoT and the Blockchain ch. 2, (29).

161 Bericht des Bundesrates, Rechtliche Grundlagen für Distributed Ledger-Technologie und Blockchain in der Schweiz <https://bit.ly/ 3DOlpV1> accessed on April 12, 2021.

162 One solution to overcome this trilemma is sharding, a technique to split up block verification and thus accelerate the process., Buterin, Why sharding is great: demystifying the technical properties (2021) <https://vitalik.ca/general/2021/04/07/sharding.html> accessed on April 12, 2021.

163 The blockchain community exchanges an enormous amount of information (open access) through platforms such as GitHub.

164 Possible solutions to the high volatility of their cryptocurrency are stablecoins that are bound to a fiat currency, e.g. Dollar. Furthermore, a smart contract can be programmed to hedge against the volatility of the respective cryptocurrency; Buterin (2013). op. cit., 12. 
access an IP right), the costs for (IP) blockchain applications ${ }^{165}$, energy consumption ${ }^{166}$ and the lack of speed of transactions ${ }^{167}$.

In general, DLTs are seen as less vulnerable to certain security risks compared to centralised solutions, such as regular data servers, because it is not sufficient to hack only one computer on the network. Public blockchains such as Bitcoin or Ethereum with a huge decentralised network can generally only be hacked if someone has the majority of the computing power (proof of work) or more than half of the value of the cryptocurrency (proof of stake) ${ }^{168}$ of the network, in what would be termed a "51\% attack".

Another security issue can be seen in smart contracts, as exposed by the DAO attack on Ethereum in 2016, when a combination of vulnerabilities in the DAO smart contract code enabled the exploitation of 3.6 million Ether. ${ }^{169}$ The solution of the Ethereum network was a hard fork ${ }^{170}$ to reverse the attacker's transactions. This was a major intervention in the original protocol, as the smart contract was supposed to be governed by the law of the blockchain but was then revised to repel this malicious attack.

Furthermore, blockchain platforms have to safeguard their cryptography themselves so that it cannot be hacked due to an old algorithm. Challenges to secure information on blockchain will increase with the development of quantum computers. The respective platforms will have to adapt their standards to post-quantum cryptography. ${ }^{171}$

This reveals how the trustworthiness of a blockchain platform depends on its security. Although initial standard guidelines have been published, specific security standards are still under development. ${ }^{172}$ Thus, the increasing offers on the market have to be examined carefully.

\subsection{Programming Issues}

Although all blockchains have the same basic function of adding blocks by a consensus mechanism, the protocols of blockchain applications can vary a lot. Common programming issues include the (lack) of Turing-completeness of a blockchain that determine the programming possibilities of smart contracts for IP; the interoperability with other (IP) platforms and online applications; as well as a reliable connection of IP "off-chain" with "on-chain" facts.

\section{a. Turing-Completeness and Programming Errors}

The Bitcoin protocol was, for instance, programmed Turing-incomplete to avoid infinite loops, i.e., program commands that repeat themselves endless times and therefore can spam the blockchain with transactions. The consequence of this limitation is that certain programming commands cannot be executed. ${ }^{173}$

Besides pre-programming the termination of the smart contract, it is possible to implement a code that allows "hacking" of the smart contract (which then implies the challenge of preventing a malicious hacker from using this "back

165 The development of blockchain applications or applications for creating your own blockchain is extensive. If an off-chain status is represented on the blockchain, further costs are incurred for tokenising goods and safeguarding their true status on the DLT.

166 In particular, the Bitcoin blockchain is criticised for its high energy consumption, because of the computational task that has to be fulfilled to mine Bitcoin. Incentivised by Bitcoin`s value, entire mining farms were developed. Nonetheless, there are blockchain networks that have established other faster and less energy-consuming versions of consensus mechanisms.

167 The speed difficulty results from the consensus mechanism and the size of transactions. A solution planned by the Ethereum Network is the "sharding" of states and history, <https://docs.ethhub.io/ethereum-roadmap/ethereum-2.0/sharding/> accessed on September 14, 2020. Another option is to decrease the number of full nodes, to give regular users a light node access to use layer two protocols that are built on top of a blockchain (layer one). Because second layer protocols create a framework on top of the blockchain, it is also referred to as an off-chain or side-chain solution, Buterin (2013). op. cit., 33. The founder of Ethereum consequently sees the platform (only) as "serving as a foundational layer", ibid 34. The tricky point is that second layer solutions again create off-chain issues because there is a lack of decentralisation.

168 Kaulartz (2018), op. cit., para. 10.

169 <https://en.wikipedia.org/wiki/The_DAO_(organization)> accessed on July 20, 2021.

170 Public blockchains need the consensus of the majority of the network to change the protocol ("hard fork"). This is different for private and consortium blockchains, which depend on the trust of the responsible institution(s), because of their power to change the blockchain.

171 <https://edps.europa.eu/data-protection/our-work/publications/techdispatch/techdispatch-22020-quantu m-computing-and_en> accessed on July 20, 2021.

172 <www.iso.org/committee/6266604/x/catalogue/>; <www.beuth.de/en/technical-rule/din-spec-3103/306199037>; other countries are also working on publishing blockchain standards, e.g. Brazil, see <https://bit.ly/36cCEPI>. Furthermore, IEEE has published standards for blockchain, see <https://blockchain.ieee.org/standards>; <www.iso.org/committee/6266604/x/catalogue/p/0/u/1/w/0/ $\mathrm{d} / 0>$ accessed on July 20, 2021.

173 A solution was introduced by Buterin in 2013 for the Ethereum platform: To pay per bytes of the transaction a specific transaction fee (called gas price). The user can either limit the transaction or set a limit for the gas price. The halting problem (infinite loop) is thus solved by the endlessness of fees for the respective transactions, Buterin (2013). op. cit., 12 . 
door"). Nevertheless, any error in the underlying code of a smart contract, can potentially cause great damage, despite the possibilities of limiting these programming risks.

Additional restrictions may arise from smart contracts themselves. They are either executed when the programmed conditions are met, or they are not executed. A smart contract itself is not able to understand the spirit of a contract and therefore intermediaries—depending on the technological development—will still be needed in this respect ${ }^{174}$.

\section{b) Interoperability with Other Platforms and Online Applications}

As the number of blockchains is growing rapidly, the interoperability with other platforms is becoming more and more important. The majority of experts invited to the WIPO standard workshop in 2019 predicted a large number of blockchain networks also for IP applications which will be able to collaborate. ${ }^{175}$ This means IP blockchains should have the ability to connect with different data systems to access, exchange, and cooperatively use data, ideally not limited to any organisation or (decentralised) platform. ${ }^{176}$ Generally, a decentralised verifiable identifier is regarded as "a key for the IP community to move forward to a digital IP ecosystem" "177 that is decisive for a trustable interaction on different (IP office) platforms.

\subsection{Data Protection and Reliability}

Various data protection issues have to be considered. Because of its immutability, data, once on the blockchain, generally cannot be altered or deleted. This appending only to the ledger contradicts the right to rectification and erasure in Arts. 16, 17 GDPR.

In the event of a permissionless blockchain, the whole ledger is saved on every computer participating as a full node and its transactions are open to everyone. Even if users change their blockchain address, a transaction can be potentially traced back years later, if for example a user attended a specific event in the real world. ${ }^{178}$ Any data that can be encrypted is also only seen in pseudonymised form. Thus, it may still count as personal data according to Art. 4 (5) and Recital 26 GDPR.

In addition, it can be a challenge to identify the responsible data controller (Art. 4 (7) GDPR) and processor (Art. 4 (8) GDPR) depending on whether a public, consortium or private blockchain is used. In a case-by-case analysis, the purposes and means of processing personal data have to be determined as well as the legal status of the different participants (full or light nodes, miners, oracles, developers or users) ${ }^{179}$. Last but not least, it can be difficult to locate the applicable data protection provisions in the case of public blockchains, because the network can potentially be joined from all over the world.

The EUIPO's suggestion is to manage most data in traditional databases and to anchor the data in a blockchain only ${ }^{180}$ ("off-chain, on-chain" model) ${ }^{181}$. Initial standards for data protection have been published by ISO. ${ }^{182}$

Moreover, even if personal data is kept off-chain in the case of a public blockchain, company data could be tracked. Once a company's address is known, data such as transaction volume-depending on the extent of the data deployed on a blockchain — could be potentially tracked by anyone, including competitors.

\subsection{Regulation and Standards}

Regulatory awareness concerning blockchain and smart contract regulation is increasing. Estonia started to test blockchain back in 2008 and has been using the technology for judicial, health and commercial registries since 2012; ${ }^{183}$ the Republic of Belarus legalised smart contracts in 2017; various EU countries have introduced regulations for cryptocurrencies; about 44 jurisdictions worldwide have announced the establishment of security frameworks, including France Germany,

174 Stefan Marschall (2020). Concept for a Multinational Patent Search using Elements of Blockchain Technology. GRUR Int., 225.

175 <www.wipo.int/meetings/en/details.jsp?meeting_id=51407> accessed 20 July 2021.

176 Bambara/Allen et al. (2019). op. cit., ch. 1, (14). API's allow a blockchain to be connected with an off-chain database.

177 WIPO Report by the Blockchain Task Force (November 2020) 3 <www.wipo.int/edocs/mdocs/cws/en/cws_8/cws_8_15-main1.pdf> accessed on July 20, 2021.

178 <http://zerocash-project.org/q_and_a\#why-do-i-need-fungibility> accessed on July 20, 2021.

179 Matthias Artzt (2021). Identifying Controllers and Processors in a Blockchain Environment in the Light of GDPR. International In-house Counsel Journal, 13(50), 2 ff. <https://iicj.net/library/detail?key=1327> accessed on July $20,2021$.

180 EC and EUIPO (2019). Anti-Counterfeiting Blockchain Use Case <https://euipo.europa.eu/ohimportal/en/web/observatory/ blockathon> accessed on July 20, 2021

181 De Meijer (2018). Blockchain Versus GDPR and Who Should Adjust Most <https://bit.ly/3oYSNRv> accessed on July 20 , 2021.

$182<$ www.iso.org/standard/75061.html?browse=tc> accessed on July 20, 2021.

183 <https://e-estonia.com/solutions/security-and-safety/e-law/> accessed on July 20, 2021. 
Italy, Sweden, Switzerland, Canada and USA; ${ }^{184}$ the EU Commission has proposed a regulatory framework on cryptoassets; ${ }^{185}$ WIPO is working on blockchain standards for IP applications; among others, ISO, IEEE and Standard Australia have published initial blockchain standards; ${ }^{186}$ China's Supreme Court recognised Blockchain as proof before court in 2018 and already uses blockchain in court proceedings itself; ${ }^{187}$ In Brazil, the first court has also accepted blockchain proof. ${ }^{188}$ In addition, the establishment of an international arbitration authority is currently being discussed. ${ }^{189}$

However, due to the inconsistent legal situation, there is clustering in individual countries. For instance, a progressive - sometimes criticised as overregulating ${ }^{190}$ _ approach to classify tokens as rights has been taken in the Liechtenstein Token Act ("Token- und VT-Dienstleister-Gesetz; TVTG")191. The Liechtenstein Act sees tokens as rights or "containers" of rights (Art. 2(1)c), Art. 7). According to the law, a "token" is a container that embodies certain rights. Hence, a token can, for example, represent (intellectual) property or other rights. According to the Liechtenstein law, the legal owner of the token also has ownership of the tokenised right. Furthermore, the law stipulates bona fide purchase Art. 5(3) and Provisions of the law of enrichment (Art. 6(3)).

Unlike the Liechtenstein Token Act, the new German law on the introduction of electronic securities ("eWpG") considers security tokens as "things" according to Section 90 BGB (Section 2(3) eWpG) ${ }^{192}$. Accordingly, § $25 \mathrm{eWpG}$ stipulates the transfer of ownership according to property law principles. ${ }^{193}$ Thus, a further step could be to discuss a possible analogy of this provision for particular non-fungible tokens besides financial instruments. NFTs representing intellectual property as "things" is already being discussed de lege ferenda ${ }^{194}$.

A holistic approach such as that adopted in Liechtenstein is currently missing in Germany. On the supranational level, provisions such as the EU directive on security tokens, in particular the directive 2014/65/EU (MIFID II) ${ }^{195}$, and also the Proposal for a Regulation on Markets in Crypto-assets (MICAR) by the European Commission ${ }^{196}$ seek to regulate the issuing of tokens and their responsibility.

$184<$ https://bit.ly/38e5MJ1, https://gbbcouncil.org/gsmi/> accessed on July 20, 2021.

$185<$ https://ec.europa.eu/commission/presscorner/detail/en/IP_20_1684> accessed on July 20, 2021.

186 <www.iso.org/committee/6266604.html> accessed accessed 20 July 2021; https://bit.ly/2I49IRI accessed on July 20, 2021; <www3.weforum.org/docs/WEF_GSMI_Technical_Standards_2020.pdf > accessed on July 20, 2021.

187 <http://english.court.gov.cn/2019-12/05/content_37527948.htm〉, 〈https://bit.ly/2Gw4gqf〉, 〈https://bit.ly/36bN02e〉 accessed on July 20, 2021.

188 <https://bit.ly/2GvxK7E> accessed on July 20, 2021.

189 Blockchain Strategy of the German Government (2019) para. 3.6 p. 13, 23 <www.blockchain-strategie.de/BC/Navigation/DE/ Home/home.html> accessed on July 20, 2021.

190 Anthony Day, S2E6 - The Roast of Non-Fungible Tokens (The Good and Very Bad of NFTs), Blockchain Won't Save the World (April 24, 2021) <https://podcasts.apple.com/ie/podcast/s2e6-roast-non-fungible-tokens-good-very-bad-nfts/ id1502561115? i=100051 8408246> accessed on April 27, 2021.

$191<$ <ww.gesetze.li/konso/2019301000> accessed accessed on July 20, 2021.

192 <https://www.bmjv.de/SharedDocs/Gesetzgebungsverfahren/Dokumente/RefE_Einfuehrung_elektr_Wertpapiere.pdf?_-_ blob= publicationFile \&v=1> accessed on July 20, 2021.

193 Partially equivalent to $\$ 929$ BGB.

194 Joshua Fairfield (2021). Tokenized: The Law of Non-Fungible Tokens and Unique Digital Property. <https://papers.ssrn.com/sol3/ papers.cfm?abstract_id=3821102> accessed on April 12, 2021; Bericht des [Schweizer] Bundesrates, Rechtliche Grundlagen für Distributed Ledger-Technologie und Blockchain in der Schweiz 〈www.newsd.admin.ch/newsd/message/attachments/55150.pdf〉 accessed on April 12, 2021, with further references. It is argued that tokens can be exclusively controlled with the control of the private key and that the decentralized, public register also creates publicity. Nonetheless, at least according to German, Swiss and Austrian law-it has to be noted that full publicity could only be achieved through public registers, which is not the case with all blockchains. The Swiss Parliament on the other hand stated that "since tokens are not physical objects, they cannot be the subject of a property right”, Bericht des [Schweizer] Bundesrates, Rechtliche Grundlagen für Distributed Ledger-Technologie und Blockchain in der Schweiz <www.newsd.admin.ch/newsd/message/attachments/55150.pdf> accessed on April 12, 2021. Interestingly enough, in cases where rights in rem exist through indirect possession and contractual agreement between immediate owner and owner, a representation of these rights in a decentralised register, such as a blockchain, might serve as medium for a legal transaction of an offchain good. A token transfer can represent a disposal of the art (that is represented by the token) by Alice. This way to transfer the ownership to Bob, Alice does not need to move the art that could be located in a third parties gallery. The art can of course be replaced by any other IP good and the gallery can be any shop, storage or other place in the world. The smart contract could transfer the token to Bob if certain conditions are met or reverse it to Alice in case of a certain event. Under German Civil Code (BGB) it could be a transaction according to $\S \S 929,930$ or $\S \S 929,931$ BGB. This is especially interesting if multi-parties are involved. Complex transactions can be easily represented by a token without the need of moving physical assets. These transactions can be realised without any paperwork and are reliable traceable in the ledger of the respective blockchain.

195 <https://eur-lex.europa.eu/legal-content/EN/TXT/?uri=CELEX\%3A32014L0065> accessed on April 12, 2021.

196 European Commission's proposal for a Regulation on Markets in Crypto-assets (MiCAR) < https://eur-lex.europa.eu/legal-content/ EN/TXT/?uri=CELEX\%3A52020PC0593> accessed on July 20, 2021. 
It can be expected that more and more countries with an awareness of the relevance of the crypto market will soon follow with regulations. Thus, every legal entity working on crypto-asset projects would be well advised to monitor the legal development and to evaluate their offers before they launch them on the market.

\section{Conclusion}

While the largely open source working blockchain community is convinced of the disruptive character of blockchain and that every marketplace in the future will incorporate the technology ${ }^{197}$, others still see blockchain-based (IP) applications as a mere hype that is characterised by buzzwords and empty promises ${ }^{198}$. The above mentioned projects show that many players in the IP ecosystem prove otherwise.

To use blockchain for IP registration, IP offices have already started to create the respective infrastructure. The standards announced by the WIPO can serve as a unifier among offices to pave the way for the legal, administrative and technical interoperability required to ensure a blockchain-enabled IP eco-system. ${ }^{199}$ In particular, manageable applications both for IP holders and for consumers will be required to enable use by a broad public. Therefore, the institutions involved, in particular IP offices, need to interact and collaborate. Otherwise, the sector will be characterised and hampered by scattered blockchain platforms. Thus, the next few years will be decisive both for the scalability and the acceptance of blockchain-based IP applications. Recent developments concerning blockchain and $\mathrm{AI}^{200}$ might accelerate this transformation.

197 Orna Ross et al. (2021). Alliance of Independent Authors (ALLI) Whitepaper <www.allianceindependentauthors.org/wp-content/ uploads/2017/07/Authors-and-the-Blockchain_Towards-a-Creator-Centered-Business-Model.pdf > accessed on July $20,2021$.

198 <www.theguardian.com/technology/2018/jan/30/blockchain-buzzword-hype-open-source-ledger-bitcoin> accessed on July $20,2021$.

199 WIPO PP "Blockchain Whitepaper Project" (2020) 4 ff. <www.wipo.int/meetings/en/details.jsp? meeting_id=57568> accessed on July 20, 2021.

200 Recently, Artificial Intelligence and Blockchain Technology funds backed by InnovFin raised a total of EUR700 mn <https:// ec.europa.eu/commission/presscorner/detail/en/ip_20_1991> accessed on July 20, 2021. 